\title{
Convergence of phase-field approximations to the Gibbs-Thomson law
}

\author{
Matthias Röger · Yoshihiro Tonegawa
}

Received: 23 March 2007 / Accepted: 11 September 2007 / Published online: 27 October 2007

C Springer-Verlag 2007

\begin{abstract}
We prove the convergence of phase-field approximations of the Gibbs-Thomson law. This establishes a relation between the first variation of the Van der Waals-CahnHilliard energy and the first variation of the area functional. We allow for folding of diffuse interfaces in the limit and the occurrence of higher-multiplicities of the limit energy measures. We show that the multiplicity does not affect the Gibbs-Thomson law and that the mean curvature vanishes where diffuse interfaces have collided. We apply our results to prove the convergence of stationary points of the Cahn-Hilliard equation to constant mean curvature surfaces and the convergence of stationary points of an energy functional that was proposed by Ohta-Kawasaki as a model for micro-phase separation in block-copolymers.
\end{abstract}

Mathematics Subject Classification (2000) Primary: 49Q20; Secondary: 35B25 - 35R35 . $80 \mathrm{~A} 22$

\section{Introduction}

Phase separation is a common phenomenon in many areas of the sciences. Alloys studied in material sciences, melting and solidification processes, or block-copolymers investigated in physical chemistry, they all show the coexistence of two or more phases, separated by thin transition layers. The main approaches to describe phase transitions are on the one hand sharp interface models and on the other hand diffuse interface models, also referred to as "phase field" or "Ginzburg-Landau" models. The relation between both kinds of models remains an outstanding question. Rigorous passages to the sharp interface limit are often difficult and generalized formulations for the limit problems are necessary to obtain the convergence of

\footnotetext{
M. Röger

Max Planck Institute for Mathematics in the Sciences, Inselstr. 22, 04103 Leipzig, Germany

e-mail: roeger@mis.mpg.de

Y. Tonegawa $(\varangle)$

Department of Mathematics, Hokkaido University Sapporo, 060-0810 Sapporo, Japan

e-mail: tonegawa@math.sci.hokudai.ac.jp
} 
diffuse approximations. However, care has to be taken that solutions satisfy the equations in a reasonably strong sense.

The goal of the present paper is to prove the convergence of diffuse approximations of the so-called Gibbs-Thomson law, which states that the mean curvature of the phase boundary is given as the trace of a function in the bulk. Our result relates the first variation of the Van der Waals-Cahn-Hilliard energy, which is the common root of most phase field models, to the first variation of the area functional. To the best of our knowledge, we give the first satisfactory solution in the case that diffuse interfaces collapse or cancel each other in the limit.

Before stating the main result we describe the setting and background of the problem.

\subsection{Phase fields, sharp interfaces, and the Gibbs-Thomson law}

The diffuse interface approach is based on a free energy that acts on smooth phase fields and that was proposed by Van der Waals [33] and later Cahn-Hilliard [7]. In a normalized form this energy is given by

$$
E_{\varepsilon}(u):=\int_{\Omega}\left(\frac{\varepsilon}{2}|\nabla u|^{2}+\frac{1}{\varepsilon} W(u)\right) d x,
$$

where $\varepsilon>0$ is a small parameter and $W$ is a nonnegative 'double-well potential' with value zero if and only if $u= \pm 1$. Domains where $u \approx 1$ or $u \approx-1$ represent two coexisting phases, separated by diffuse interfaces. Formal arguments show that $E_{\varepsilon}$ favors transition layers with a thickness of order $\varepsilon$. Hence, as $\varepsilon$ tends to zero the diffuse interfaces become sharp.

One naturally associated quantity to the Cahn-Hilliard energy is its $L^{2}$-functional derivative, which often corresponds to the chemical potential,

$$
f_{\varepsilon}=-\varepsilon \Delta u+\frac{1}{\varepsilon} W^{\prime}(u) .
$$

In many applications $f_{\varepsilon}$ is given by means of other quantities and a certain control on $f_{\varepsilon}$ is available. The corresponding functional derivative of the surface area functional, evaluated at a smooth compact hypersurface $\Sigma$, is given by the mean curvature of $\Sigma$ and (1.2) formally corresponds to the following equation, in solidification processes known as Gibbs-Thomson law (and we will adopt this term throughout the paper),

$$
H=\sigma f,
$$

with a surface tension coefficient $\sigma>0$. The Gibbs-Thomson law relates the local geometry of the phase boundary to a function $f: \Omega \rightarrow \mathbb{R}$ in the bulk, for example the temperature or the chemical potential.

\subsection{Main results}

Let us first state our main result in a concise form (we will prove a slightly stronger statement, given in Theorem 3.2).

Theorem 1.1 Suppose $p>n$ and let sequences of functions $\left\{u_{\varepsilon}\right\}_{\varepsilon>0} \subset W^{3, p}(\Omega)$ and functions $\left\{f_{\varepsilon}\right\}_{\varepsilon>0} \subset W^{1, p}(\Omega)$ be given such that (1.2) holds and such that

$$
\begin{aligned}
E_{\varepsilon}\left(u_{\varepsilon}\right) & \leq \Lambda, \\
u_{\varepsilon} & \rightarrow u \text { in } L^{1}(\Omega), \\
f_{\varepsilon} & \rightarrow f \text { weakly in } W^{1, p}(\Omega)
\end{aligned}
$$


as $\varepsilon \rightarrow 0$. Then $u$ is of bounded variation and takes only values in $\{-1,1\}$. Moreover there exists a unique generalized mean curvature $H$ of the 'phase boundary' $\Sigma:=\Omega \cap \partial^{*}\{u=1\}$ and

$$
\sigma H=f \text { holds } \mathcal{H}^{n-1}-\text { almost everywhere on } \Sigma \text {. }
$$

Here $\sigma=\int_{-1}^{1} \sqrt{W(s) / 2} d s$ is the surface tension coefficient and the sign of $H$ is chosen positive for spherical $\{u=1\}$.

This Theorem uses generalized formulations for the phase boundary and the mean curvature. The notion of mean curvature is based on a measure-theoretic approach and was introduced by the first author in [31]. We refer to the Appendix 7.2 for the exact definition.

Remark 1.2 For a sequence $\left(u_{\varepsilon}\right)_{\varepsilon>0}$ that satisfies the uniform energy bound (1.4) and a sequence $\left(f_{\varepsilon}\right)_{\varepsilon>0}$ that is uniformly bounded in $W^{1, p}(\Omega)$ there exists a subsequence $\varepsilon \rightarrow 0$ such that (1.5), (1.6) hold. Besides these uniform bounds no other conditions, such as energy minimality, are required to apply Theorem 1.1. For this reason the result is relevant to a large class of stationary and time-dependent problems. In Sect. 7 we use our results to characterize the limit of stationary points of the Cahn-Hilliard functional (1.1) and to prove the convergence of stationary points in a model for block-copolymers.

The assumption (1.6) on the chemical potentials $f_{\varepsilon}$ is still restrictive. We conjecture that the (weak) convergence of $f_{\varepsilon}$ in $W^{1, p}(\Omega)$ with $p>n / 2$ would suffice to conclude (1.7). However, our techniques yet require the continuity of $f$, which is ensured only if $p>n$. For the Cahn-Hilliard equation for example the natural regularity of the chemical potential is $W^{1,2}(\Omega)$ in space. Hence, our result does not apply in this case.

\subsection{Related results and main techniques}

Since the fundamental work of Modica and Mortola [21,22] on the convergence of $E_{\varepsilon}$ to the area functional the relation between their first variations has drawn attention. Modica [21] and Sternberg [40] proved that minimizers of $E_{\varepsilon}$ under a volume constraint converge to areaminimizing hypersurfaces with an integral constraint. Luckhaus-Modica [19] then showed that the Lagrange-multipliers associated with the volume constraint converge to the constant mean curvature of the limiting hypersurface. Ilmanen [17] considered the corresponding $L^{2}$-gradient flows and proved the convergence of the Allen-Cahn equation to the meancurvature flow, in the varifold formulation of Brakke [5]. Convergence of various other phase field problems to the corresponding sharp interface models have been shown either formally or rigorously $[1,6,8,9,18,27,38]$, sometimes in quite involved weak formulations.

The second author considered, partly in joint-work with Hutchinson, the convergence of diffuse interface approximations of the Gibbs-Thomson law, under different assumptions on the chemical potential $[16,41,42]$. However, the Gibbs-Thomson relation is only verified in an (in some respect unsatisfactory) multiplicity-dependent formulation, see (1.9) and the discussion below.

Schätzle [35] considered a sequence of hypersurfaces with mean curvature given by a Sobolev function in the ambient space and obtained that the Gibbs-Thomson law holds in the limit in a rather clean varifold formulation. In [35] the chemical potentials $f_{\varepsilon}$ in (1.6) need only to converge in a Sobolev space $f \in W^{1, p}(\Omega)$ with $p>n / 2$, c.f. Remark 1.2.

Geometric Measure Theory provides suitable generalized formulations in spaces that allow for the compactness of approximations. Luckhaus-Modica [19] and LuckhausSturzenhecker [20] introduced a weak formulation of the Gibbs-Thomson law (1.3) for characteristic functions of bounded variation. This formulation is rather natural and has the 
advantage of being based directly on the phase function. However, justifying the GibbsThomson law in the limit of approximations requires the additional assumption that no cancelation of (diffuse) interfaces occurs. Unfortunately, this property does in general not hold [34].

To master such cancelations Ilmanen [17] used a varifold-approach. He considered the limit of the diffuse surface-area measures (energy measures)

$$
\mu_{\varepsilon}:=\left(\frac{\varepsilon}{2}\left|\nabla u_{\varepsilon}\right|^{2}+\frac{1}{\varepsilon} W\left(u_{\varepsilon}\right)\right) \mathcal{L}^{n} .
$$

The idea behind is that this limit makes information visible that is lost in the limit of the phase fields: Where cancelation of the approximate phase boundaries occurs the limit $\mu$ of the measures $\mu_{\varepsilon}$ carries a higher multiplicity. The support of $\mu$ eventually extends the limit phase boundary by hidden boundaries. Showing that the limit measure is in fact given as a integer-rectifiable varifold with a weak mean curvature vector, the Gibbs-Thomson law can be verified in a varifold formulation.

This strategy was used for various problems by Chen [9], Soner [39], HutchinsonTonegawa [16], Tonegawa [41,42], and others. However, in none of these papers the problem of higher multiplicity was completely solved. Typically the convergence of the diffuse phase fields and the diffuse surface-area measures $\mu_{\varepsilon}$ is shown and the rectifiability of the limit $\mu$ as well as the existence of a weak mean curvature $H_{\mu}$ is obtained. Still, the Gibbs-Thomson law holds only in a multiplicity-dependent formulation

$$
H_{\mu}=(N \sigma)^{-1} f \quad \mathcal{H}^{n-1} \text {-almost everywhere on } \partial^{*}\{u=1\},
$$

where $N$ is the density function of the measure $\mu$. This formulation is for two reasons unsatisfactory: First the Gibbs-Thomson law should be satisfied by the phase boundary rather than by the (in view of the applications) 'obscure' measure $\mu$. Secondly, the density function $N$ should not affect the Gibbs-Thomson law. To prove the full result (1.9) has to be complemented by

$$
f=H_{\mu}=0 \quad \mathcal{H}^{n-1} \text {-almost everywhere on } \partial^{*}\{u=1\} \cap\{N>1\} .
$$

In recent years progress has been made on this issue. Schätzle [35] proves the Gibbs-Thomson law in the limit of an approximation by hypersurfaces: There the weak mean curvature $H_{\mu}$ of the limit measure $\mu$ satisfies (1.3) and (1.10). It was then shown by the first author [31] that $H_{\mu}$ is in fact a property of the phase boundary $\partial^{*}\{u=1\}$, see Appendix 7.2. This is crucial in order to apply the (stationary) convergence result [35] to evolution problems [31,32].

The higher-multiplicity problem is even more challenging in the context of the sharp interface limit of diffuse approximations, due to the singular nature of this limit process. The three main ingredients of our proof are first an earlier result of the second author [42] on the convergence of certain phase field equations with chemical potential. This ensures rectifiability, existence of a weak mean curvature with appropriate regularity and the multiplicitydependent Gibbs-Thomson relation (1.9). The second ingredient is the fine local analysis of Schätzle [36] on integer-rectifiable varifolds with sufficiently regular weak mean curvature. The third important argument is a comparison principle for the phase fields $u_{\varepsilon}$ and diffuse approximations of suitably constructed comparison graphs.

\subsection{Organization of the paper}

In the next section we will precisely formulate our assumptions and introduce some notations. Section 3 states our main result. A localization step in Sect. 4 prepares a contradiction 
argument that we will use in Sect. 5 to prove our main Theorem 3.2. We first assume that a certain comparison principle, which is given in Proposition 6.11, holds. Section 6 is then devoted to the proof of this Proposition. Finally we give in Sect. 7 two applications of Theorem 1.1 and we recall in the appendix the definition of a generalized mean curvature for phase boundaries that we will use.

\section{Notations and assumptions}

We state first all assumptions and definitions, including those already appeared in the introduction.

Assumption 2.1 Consider a bounded domain $\Omega \subset \mathbb{R}^{n}$ with Lipschitz-boundary and the standard quartic double-well potential $W$ given by

$$
W(r):=\frac{1}{4}\left(1-r^{2}\right)^{2} .
$$

We define an energy functional $E_{\varepsilon}$ on $W^{1,2}(\Omega)$,

$$
E_{\varepsilon}(u):=\int_{\Omega}\left(\frac{\varepsilon}{2}|\nabla u|^{2}+\frac{1}{\varepsilon} W(u)\right) d x .
$$

Suppose $p>n$ and let sequences $\left(u_{\varepsilon}\right)_{\varepsilon>0} \subset W^{3, p}(\Omega)$ and $\left(f_{\varepsilon}\right)_{\varepsilon>0} \subset W^{1, p}(\Omega)$ be given such that

$$
\begin{aligned}
E_{\varepsilon}\left(u_{\varepsilon}\right) \leq \Lambda & \text { for all } \varepsilon>0, \\
& -\varepsilon \Delta u_{\varepsilon}+\frac{1}{\varepsilon} W^{\prime}\left(u_{\varepsilon}\right)=f_{\varepsilon} \quad \text { in } \Omega .
\end{aligned}
$$

Assume further that

$$
\begin{aligned}
& u_{\varepsilon} \rightarrow u \quad \text { in } L^{1}(\Omega), \quad u \in B V(\Omega,\{-1,1\}), \\
& f_{\varepsilon} \rightarrow f \quad \text { weakly in } W^{1, p}(\Omega) .
\end{aligned}
$$

We may generalize $W$ to be any $C^{3}$-function with two non-degenerate minima and one local maximum, so that the results in [42] apply.

We next associate diffuse surface-area measures and appropriate varifolds to the functions $u_{\varepsilon}$.

Definition 2.2 For $u_{\varepsilon}$ we define Radon-measures $\mu_{\varepsilon}$ on $\Omega$,

$$
\mu_{\varepsilon}:=\left(\frac{\varepsilon}{2}\left|\nabla u_{\varepsilon}\right|^{2}+\frac{1}{\varepsilon} W\left(u_{\varepsilon}\right)\right) \mathcal{L}^{n}
$$

and $(n-1)$-varifolds $V_{\varepsilon}$ on $G^{n-1}(\Omega):=\Omega \times G(n, n-1)$,

$$
V_{\varepsilon}(\zeta)=\int_{G^{n-1}(\Omega)} \zeta(x, S) d V_{\varepsilon}(x, S):=\int_{\Omega} \zeta\left(x, v_{\varepsilon}^{\perp}(x)\right) d \mu_{\varepsilon}(x)
$$

for all $\zeta \in C_{c}^{0}\left(G^{n-1}(\Omega)\right)$, where

$$
v_{\varepsilon}:=\frac{\nabla u_{\varepsilon}}{\left|\nabla u_{\varepsilon}\right|} \quad \text { if } \nabla u_{\varepsilon} \neq 0, \quad v_{\varepsilon}=(1,0, \ldots, 0)^{T} \quad \text { otherwise. }
$$


Remark 2.3 By the Sobolev embedding Theorem, (2.5), and $p>n$ it follows that

$$
\begin{aligned}
& f_{\varepsilon}, f \in C^{0, \alpha}(\bar{\Omega}), \\
& f_{\varepsilon} \rightarrow f \quad \text { in } C^{0, \beta}(\bar{\Omega})
\end{aligned}
$$

for $\alpha:=1-n / p$ and all $0 \leq \beta<\alpha$.

Moreover, by (2.2) there exists a subsequences $\varepsilon \rightarrow 0$ and a Radon-measure $\mu$ on $\Omega$ such that

$$
\mu_{\varepsilon} \rightarrow \mu \text { as Radon-measures on } \Omega \text {. }
$$

Here and in the following we often do not relabel subsequences. In particular we assume from now on that (2.10) holds for the whole sequence $\varepsilon \rightarrow 0$.

Finally we define the mean-curvature operator $\mathrm{H}$ for graphs: for $p \in \mathbb{R}^{n-1}, X \in \mathcal{S}(n-1)$, where $\mathcal{S}(n-1)$ denotes the space of symmetric matrices in $\mathbb{R}^{(n-1) \times(n-1)}$, set

$$
\mathrm{H}(p, X):=\left(1+|p|^{2}\right)^{-\frac{3}{2}}\left(\operatorname{tr} X+|p|^{2}\left(I d-\frac{p}{|p|} \otimes \frac{p}{|p|}\right): X\right) .
$$

\section{Statement of results}

The first conclusion we draw is a direct consequence of previous results of the second author.

Theorem 3.1 [42] Let Assumption 2.1 hold, let $\mu$ satisfy (2.10), and set

$$
\sigma:=\int_{-1}^{1} \sqrt{\frac{1}{2} W(s)} d s .
$$

Then $(2 \sigma)^{-1} \mu$ is $(n-1)$-integer-rectifiable,

$$
\theta^{(n-1)}(\mu, \cdot)=N(\cdot) 2 \sigma, \quad N \text { integer-valued } .
$$

Moreover $\mu$ has weak mean curvature $H_{\mu} \in L^{\infty}(\mu)$, and

$$
H_{\mu}=\frac{f}{N(\cdot) \sigma} v
$$

holds $\mu$-almost everywhere, where $v=\frac{\nabla u}{|\nabla u|}$ on $\partial^{*}\{u=1\}$ and $v=0$ elsewhere.

Proof See [42].

We remark that in [42] only $p>n / 2$ is assumed. Our main results are summarized in the following theorem.

Theorem 3.2 Let Assumption 2.1 hold, let $\mu$ satisfy (2.10), and let $N$ denote the multiplicity function of $\mu$ as in Theorem 3.1. Then

(1) $\mu$-almost everywhere in $\{N(\cdot) \geq 3$ odd $\}$

$$
H_{\mu}=0, \quad f=0 .
$$


(2) $\mu$-almost everywhere in $\{N(\cdot) \geq 2$ even $\}$

$$
H_{\mu}=0, \quad f \leq 0 \text { in }\{u=1\} \cap \operatorname{supp}(\mu), \quad f \geq 0 \text { in }\{u=-1\} \cap \operatorname{supp}(\mu) .
$$

(3) the function $H: \partial^{*}\{u=1\} \rightarrow \mathbb{R}$ defined as

$$
H:=H_{\mu} \cdot \frac{\nabla u}{|\nabla u|}
$$

is the generalized mean curvature of $\partial^{*}\{u=1\}$ in the sense of Definition A.2.

(4) Finally

$$
\sigma H=f
$$

holds $\mathcal{H}^{n-1}$-almost everywhere on $\partial^{*}\{u=1\} \cap \Omega$.

\section{Localization}

In this section we show that we can restrict ourselves to a "generic" local situation, where the support of $\mu$ is well described in terms of graphs. We then apply a result of Schätzle [36] that gives a fine description of the varifold $\mu$.

Lemma 4.1 It is sufficient to prove (3.3), (3.4) for $\mu$-almost all generic points, that are those points $x_{0} \in \Omega$ satisfying

$$
\begin{aligned}
& T_{x_{0}} \mu \text { exists, } \\
& N_{0}:=N\left(x_{0}\right) \in \mathbb{N}, \\
& \theta\left(\mu,\left\{N(\cdot)=N_{0}\right\}, x_{0}\right)=1 .
\end{aligned}
$$

In addition we may assume without loss of generality that

$$
\begin{aligned}
& \begin{cases}x_{0} \in \operatorname{supp}(\mu) \cap \partial^{*}\{u=1\} & \text { if } N\left(x_{0}\right) \text { is odd, } \\
x_{0} \in \operatorname{supp}(\mu) \backslash \partial^{*}\{u=1\} & \text { if } N\left(x_{0}\right) \text { is even, }\end{cases} \\
& \lim _{\varrho \rightarrow 0}\left(\sup \left\{\varrho^{-1} \operatorname{dist}\left(x, T_{x_{0}} \mu\right): x \in \operatorname{supp}(\mu) \cap B_{\varrho}^{n}\left(x_{0}\right)\right\}\right)=0 .
\end{aligned}
$$

Proof We show that (4.1)-(4.5) hold $\mu$-almost everywhere in $\{N(\cdot) \geq 2\}$.

Since $\mu$ is integer-rectifiable (4.1), (4.2) are satisfied $\mu$-almost everywhere. Since the set $\left\{\theta^{n-1}(\mu, \cdot)=N_{0}\right\}$ is $\mu$-measurable (4.3) holds for $\mu$-almost all points. By [42, Theorem 2.2] $\mathcal{H}^{n-1}$-almost all $x \in \operatorname{supp}(\mu)$ with odd density $N(x)$ belong to $\partial^{*}\{u=1\}$ and $\mu$-almost all points with even density belong to $\operatorname{supp}(\mu) \backslash \partial^{*}\{u=1\}$. Finally (4.5) follows from [37, Lemma 17.11].

We fix $x_{0}$ such that (4.1)-(4.5) hold. After applying a suitable translation and rotation we may assume that $x_{0}=0$ and

$$
T_{0} \mu=\mathbb{R}^{n-1} \times\{0\} .
$$

To apply a contradiction argument we assume (3.3), (3.4) to be false.

Assumption 4.2 Suppose that

$$
N_{0} \geq 2 \text { and } f(0) \neq 0 .
$$


By (2.8), (4.5) there exists $\varrho_{0}>0$ such that

$$
\begin{aligned}
& |f-f(0)| \leq \frac{1}{27}|f(0)| \quad \text { on } \overline{B_{\varrho_{0}}(0)} \times\left[-5 \varrho_{0}, 5 \varrho_{0}\right], \\
& \operatorname{supp}(\mu) \cap\left(B_{\varrho_{0}}(0) \times\left(-5 \varrho_{0}, 5 \varrho_{0}\right)\right) \subset B_{\varrho_{0}}(0) \times\left(-\varrho_{0}, \varrho_{0}\right) .
\end{aligned}
$$

We distinguish four cases depending on whether $f(0)<0$ or $f(0)>0$ and whether $u=1$ or $u=-1$ in the region 'above' $\operatorname{supp}(\mu)$. In the following we consider the case that

$$
\begin{aligned}
f(0) & >0, \\
u(y, t) & =1 \quad \text { for } y \in B_{\varrho_{0}}(0), \quad t>\varrho_{0} .
\end{aligned}
$$

That implies that

$$
u(y, t)=\left\{\begin{array}{ll}
-1 & \text { if } N_{0} \text { is odd, } \\
1 & \text { if } N_{0} \text { is even }
\end{array} \quad \text { for all } y \in B_{\varrho_{0}}(0), t<-\varrho_{0} .\right.
$$

The other cases can be treated analogously or follow from a symmetry argument. By (2.9) we obtain that

$$
\left|f_{\varepsilon}-f\right| \leq \frac{1}{27} f(0) \quad \text { on } \overline{B_{\varrho_{0}}(0)} \times\left[-5 \varrho_{0}, 5 \varrho_{0}\right]
$$

for all $\varepsilon>0$ sufficiently small.

In the next step we apply a result of Schätzle [36] on the local structure of the measure $\mu$. First we need some definitions.

Definition 4.3 We define the upper and lower height-functions $\varphi_{+}, \varphi_{-}: B_{\varrho_{0}}^{n-1}(0) \rightarrow$ $[-\infty, \infty]$,

$$
\begin{aligned}
& \varphi_{+}(y):=\sup \left\{t \in\left(-5 \varrho_{0}, 5 \varrho_{0}\right):(y, t) \in \operatorname{supp}(\mu) \cap\left(B_{\varrho_{0}}^{n-1}(0) \times \mathbb{R}\right)\right\}, \\
& \varphi_{-}(y):=\inf \left\{t \in\left(-5 \varrho_{0}, 5 \varrho_{0}\right):(y, t) \in \operatorname{supp}(\mu) \cap\left(B_{\varrho_{0}}^{n-1}(0) \times \mathbb{R}\right)\right\},
\end{aligned}
$$

with the convention that the supremum over an empty set is $-\infty$ and the infimum over an empty set is $+\infty$.

Moreover we set for all $y$ with $\varphi_{+}(y)>-\infty$

$$
F_{+}(y):= \begin{cases}\frac{f\left(y, \varphi_{+}(y)\right)}{N\left(y, \varphi_{+}(y)\right) \sigma} & \text { if } N\left(y, \varphi_{+}(y)\right) \text { is odd, } \\ 0 & \text { if } N\left(y, \varphi_{+}(y)\right) \text { is even, }\end{cases}
$$

and for all $y$ with $\varphi_{-}(y)<\infty$

$$
F_{-}(y):= \begin{cases}\frac{f\left(y, \varphi_{-}(y)\right)}{N\left(y, \varphi_{-}(y)\right) \sigma} & \text { if } N\left(y, \varphi_{-}(y)\right) \text { is odd } \\ 0 & \text { if } N\left(y, \varphi_{-}(y)\right) \text { is even. }\end{cases}
$$

Proposition 4.4 The height-functions $\varphi_{ \pm}$are twice approximately differentiable $\mathcal{L}^{n-1}$ almost everywhere in $\left\{\varphi_{ \pm} \in \mathbb{R}\right\}$ with

$$
\begin{aligned}
-\mathrm{H}\left(\nabla \varphi_{+}, D^{2} \varphi_{+}\right) & =-F_{+}, \\
-\mathrm{H}\left(\nabla \varphi_{-}, D^{2} \varphi_{-}\right) & =-(-1)^{1+N_{0}} F_{-},
\end{aligned}
$$

Moreover, for all $s>n-1, s \geq 2$ the upper height-function $\varphi_{+}$is an $W^{2, s}$-viscosity subsolution of

$$
-\mathrm{H}\left(\nabla \varphi_{+}, D^{2} \varphi_{+}\right) \leq-F_{+}
$$


and the lower height-function $\varphi_{-}$is a $W^{2, s}$-viscosity supersolution of

$$
-\mathrm{H}\left(\nabla \varphi_{-}, D^{2} \varphi_{-}\right) \geq-(-1)^{1+N_{0}} F_{-}
$$

Proof Since $H_{\mu} \in L^{\infty}(\mu)$ we obtain from [36, Theorem 6.1] that

$$
H_{\mu}\left(\cdot, \varphi_{+}(\cdot)\right)=\nabla \cdot\left(\frac{\nabla \varphi_{+}}{\sqrt{1+\left|\nabla \varphi_{+}\right|^{2}}}\right) \frac{1}{\sqrt{1+\left|\nabla \varphi_{+}\right|^{2}}}\left(\begin{array}{c}
-\nabla \varphi_{+} \\
1
\end{array}\right)
$$

$\mathcal{L}^{n-1}$-almost everywhere in $\left\{\varphi_{+} \in \mathbb{R}\right\}$ and that $\varphi_{+}$is for all $s>n-1, s \geq 2$ a $W^{2, s}$-viscosity subsolution of

$$
-\nabla \cdot\left(\frac{\nabla \varphi_{+}}{\sqrt{1+\left|\nabla \varphi_{+}\right|^{2}}}\right) \leq-H_{\mu}\left(\cdot, \varphi_{+}(\cdot)\right) \frac{1}{\sqrt{1+\left|\nabla \varphi_{+}\right|^{2}}}\left(\begin{array}{c}
-\nabla \varphi_{+} \\
1
\end{array}\right) .
$$

From (3.2), (4.22) we deduce that

$$
\frac{f\left(\cdot, \varphi_{+}(\cdot)\right)}{N\left(\cdot, \varphi_{+}(\cdot)\right) \sigma} v\left(\cdot, \varphi_{+}(\cdot)\right)=\mathrm{H}\left(\nabla \varphi_{+}, D^{2} \varphi_{+}\right) \frac{1}{\sqrt{1+\left|\nabla \varphi_{+}\right|^{2}}}\left(\begin{array}{c}
-\nabla \varphi_{+} \\
1
\end{array}\right) .
$$

Next we observe that (4.11) implies

$$
v\left(y, \varphi_{+}(y)\right)=\frac{\nabla u}{|\nabla u|}\left(y, \varphi_{+}(y)\right)=\left(1+\left|\nabla \varphi_{+}\right|^{2}\right)^{-\frac{1}{2}}\left(-\nabla \varphi_{+}(y), 1\right)^{T}
$$

for $\mathcal{L}^{n-1}$ almost all $y \in\left\{\varphi_{+} \in \mathbb{R}\right\}$ such that $\left(y, \varphi_{+}(y)\right) \in \partial^{*}\{u=1\}$, and $v=0$ otherwise. By [42, Theorem 2.2] up to a $\mu$-nullset $\partial^{*}\{u=1\}=\{N \geq 1$ odd $\}$ and we obtain from (4.16) and (4.24), (4.25) that (4.18) holds. (4.20) follows by the same arguments. To obtain (4.19), (4.21) we observe that

$$
v\left(y, \varphi_{-}(y)\right)=\frac{\nabla u}{|\nabla u|}\left(y, \varphi_{-}(y)\right)=(-1)^{1+N_{0}}\left(1+\left|\nabla \varphi_{-}\right|^{2}\right)^{-\frac{1}{2}}\left(-\nabla \varphi_{-}(y), 1\right)^{T}
$$

and we proceed as above.

We choose below a "good point" for which we derive a contradiction to Assumption 4.2. Before, we need another definition.

Definition 4.5 We say that a function $\psi$ has a second-order Taylor expansion at a point $y_{1} \in \mathbb{R}^{n-1}$ if there exist $p \in \mathbb{R}^{n-1}, X \in \mathcal{S}(n-1)$ such that

$$
\lim _{\substack{y \rightarrow y_{1} \\ \psi(y) \in \mathbb{R}}}\left|y-y_{1}\right|^{-2}\left|\psi(y)-\psi\left(y_{1}\right)-p \cdot\left(y-y_{1}\right)-\frac{1}{2}\left(y-y_{1}\right) \cdot X\left(y-y_{1}\right)\right|=0 .
$$

We then set $\nabla \psi\left(y_{1}\right):=p, D^{2} \psi\left(y_{1}\right):=X$.

Lemma 4.6 There exists a point $y_{1} \in B_{\varrho_{0}}(0)$ such that

$$
\begin{aligned}
& \varphi_{+}\left(y_{1}\right)=\varphi_{-}\left(y_{1}\right), \\
& x_{1}:=\left(y_{1}, \varphi_{ \pm}\left(y_{1}\right)\right) \text { is a generic point, } \\
& \theta^{n-1}\left(\mu, x_{1}\right)=N_{0}, \\
& \varphi_{ \pm} \text {have a second-order Taylor expansion at } y_{1}, \\
& -\mathrm{H}\left(\nabla \varphi_{ \pm}\left(y_{1}\right), D^{2} \varphi_{ \pm}\left(y_{1}\right)\right)=-F_{ \pm}\left(y_{1}\right) .
\end{aligned}
$$


Proof Since the weak mean curvature $H_{\mu}$ belongs to $L^{\infty}(\mu)$ by (3.2) and since $0 \in \mathbb{R}^{n}$ is a generic point we can apply [35, Lemma 3.4] (see also Step 3 in the proof of [32, Lemma 3.2]) and obtain that the set

$$
\begin{gathered}
\Sigma_{0}:=\left\{x=\left(y, \varphi_{ \pm}(y)\right): y \in B_{\varrho_{0}}^{n-1}(0) \cap\left\{\varphi_{+}=\varphi_{-}\right\},\right. \\
\left.x \in \operatorname{supp}(\mu) \text { is generic, } N(x)=N_{0}\right\}
\end{gathered}
$$

has full density with respect to $\mu$ in $0 \in \mathbb{R}^{n}$. This property was essentially deduced from Brakke's Lipschitz Approximation Theorem. The curvature bound ensures a strong control on the approximations, see $[31,35]$ for the details. From the Coarea Formula we then deduce that (4.28)-(4.30) holds in a set with full $\mathcal{L}^{n-1}$-density in $0 \in \mathbb{R}^{n-1}$. Finally, (4.31), (4.32) are satisfied $\mathcal{L}^{n-1}$ almost everywhere in $\left\{\varphi_{+}=\varphi_{-}\right\}$by [36, Proposition 4.1] and (4.18), (4.19), where we use that $F_{ \pm}\left(y_{1}\right)=0$ if $N_{0}$ is even.

\section{Proof of Theorem 3.2}

We fix $y_{1} \in B_{\varrho_{0}}(0)$ such that (4.28)-(4.32) hold and consider the second order Taylor approximation of $\varphi_{ \pm}$at $y_{1}$,

$$
P_{1}(y):=\varphi_{ \pm}\left(y_{1}\right)+\nabla \varphi_{ \pm}\left(y_{1}\right) \cdot\left(y-y_{1}\right)+\frac{1}{2}\left(y-y_{1}\right) \cdot D^{2} \varphi_{ \pm}\left(y_{1}\right)\left(y-y_{1}\right) .
$$

From (4.31) we then deduce that

$$
\lim _{\substack{y \rightarrow y_{1} \\ \varphi \pm(y) \in \mathbb{R}}}\left|y-y_{1}\right|^{-2}\left|P_{1}(y)-\varphi_{ \pm}(y)\right|=0 .
$$

Lemma 5.1 For all $\omega>0$ there is $\varrho_{1}>0$ such that

$$
\begin{aligned}
& B_{\varrho_{1}}\left(y_{1}\right) \subset B_{\varrho_{0}}(0), \\
& \left|P_{1}(y)-\varphi_{+}(y)\right|<\omega\left|y-y_{1}\right|^{2} \text { on } \mathrm{B}_{\varrho_{1}}\left(\mathrm{y}_{1}\right) \cap\left\{\varphi_{+}>-\infty\right\}
\end{aligned}
$$

and such that for any $0<\varrho<\varrho_{1}$ there exists a unique solution $\psi \in C^{\infty}\left(\overline{B_{\varrho}\left(y_{1}\right)}\right)$ of

$$
\begin{aligned}
-\mathrm{H}\left(\nabla \psi, D^{2} \psi\right) & =-\frac{2 f\left(y_{1}\right)}{3 \sigma} \text { in } \mathrm{B}_{\varrho}\left(\mathrm{y}_{1}\right), \\
\psi(y) & =P_{1}(y)+\omega \varrho^{2} \text { on } \partial \mathrm{B}_{\varrho}\left(\mathrm{y}_{1}\right) .
\end{aligned}
$$

Proof Since $y_{1} \in B_{\varrho_{0}}(0)$ and by (5.2) for any $\varrho_{1}>0$ sufficiently small the properties (5.3), (5.4) hold. Since $P_{1}$ is smooth and since the right-hand side of equation (5.5) is constant we deduce from [13, Theorem 16.10] that for all

$$
\varrho \leq \varrho_{1}, \quad \varrho_{1}=\varrho_{1}\left(n, \sigma, f\left(y_{1}\right)\right)
$$

a unique solution $\psi \in C^{2, \gamma}\left(\overline{B_{\varrho}\left(y_{1}\right)}\right), 0<\gamma<1$, of (5.5), (5.6) exists. The higher regularity of $\psi$ follows from standard elliptic theory and the smoothness of the data in (5.5), (5.6).

The next Proposition is the heart of the contradiction argument. It relies on the fact that the approximations $u_{\varepsilon}$ behave as if the curvature of the limit interface is given by $f / \sigma$ rather than by $f /(N \sigma)$. 
Proposition 5.2 Let $\psi$ be as in Lemma 5.1. Then

$$
\psi \geq \varphi_{+} \text {in } \mathrm{B}_{\varrho}\left(\mathrm{y}_{1}\right) .
$$

The proof of this Proposition uses a comparison between $u_{\varepsilon}$ and approximations $v_{\varepsilon}$ of $2 \mathcal{X}_{E}-1$, where $E$ is the region above the graph of $\psi$. We postpone this proof to Sect. 6 and continue the proof of Theorem 3.2.

Lemma 5.3 For all $0<\omega<1$ there exists $\tilde{\varrho}_{1}>0$ such that for all $0<\varrho<\tilde{\varrho}_{1}$ the function

$$
\eta(y):=P_{1}(y)-2 \omega\left(\varrho^{2}-\left|y-y_{1}\right|^{2}\right)
$$

satisfies for all $y \in B_{\varrho}\left(y_{1}\right)$

$$
-\mathrm{H}\left(\nabla \eta, D^{2} \eta\right) \geq-F_{ \pm}\left(y_{1}\right)-5(n-1) \omega .
$$

Proof We compute that for $y \in B_{\varrho}\left(y_{1}\right)$

$$
\begin{aligned}
\left|\nabla \eta(y)-\nabla \varphi_{ \pm}\left(y_{1}\right)\right| & =\left|D^{2} \varphi_{ \pm}\left(y_{1}\right)\left(y-y_{1}\right)+4 \omega\left(y-y_{1}\right)\right| \\
& \leq\left(\left|D^{2} \varphi_{ \pm}\left(y_{1}\right)\right|+4\right) \varrho, \\
\left|D^{2} \eta(y)\right| & \leq\left|D^{2} \varphi_{ \pm}\left(y_{1}\right)\right|+4 .
\end{aligned}
$$

Hence, we can choose $\tilde{\varrho}_{1}=\tilde{\varrho}_{1}\left(\nabla \varphi_{ \pm}\left(y_{1}\right), D^{2} \varphi_{ \pm}\left(y_{1}\right), \omega\right)$ such that

$$
\left|\mathrm{H}\left(\nabla \eta, D^{2} \eta\right)-\mathrm{H}\left(\varphi_{ \pm}\left(y_{1}\right), D^{2} \eta\right)\right| \leq(n-1) \omega \text { in } B_{\varrho}\left(y_{1}\right)
$$

for all $0<\varrho<\tilde{\varrho}_{1}$. This implies that

$$
\begin{aligned}
-\mathrm{H}\left(\nabla \eta, D^{2} \eta\right) & \geq-\mathrm{H}\left(\nabla \varphi_{ \pm}\left(y_{1}\right), D^{2} \varphi_{ \pm}\left(y_{1}\right)\right)-4 \omega \mathrm{H}\left(\nabla \varphi_{ \pm}\left(y_{1}\right), I d\right)-(n-1) \omega \\
& \geq-\mathrm{H}\left(\nabla \varphi_{ \pm}\left(y_{1}\right), D^{2} \varphi_{ \pm}\left(y_{1}\right)\right)-5(n-1) \omega,
\end{aligned}
$$

where we have used that $\mathrm{H}(p, I d) \leq n-1$ for all $p \in \mathbb{R}^{n-1}$.

Proof of Theorem 3.2 Choose $0<\omega<\frac{f\left(y_{1}\right)}{30(n-1) \sigma}$ and $0<\varrho<\min \left(\varrho_{1}, \tilde{\varrho}_{1}\right)$. Let $\psi, \eta$ be the functions constructed in Lemma 5.1 and Lemma 5.3. We then obtain from (5.5), (5.9), the definition of $F_{ \pm},(4.7)$, and $f\left(y_{1}\right)>0$ that

$$
\begin{aligned}
-\mathrm{H}\left(\nabla \psi, D^{2} \psi\right)+\mathrm{H}\left(\nabla \eta, D^{2} \eta\right) & \leq-\frac{2 f\left(y_{1}\right)}{3 \sigma}+\frac{f\left(y_{1}\right)}{N\left(y_{1}, \varphi_{ \pm}\left(y_{1}\right)\right) \sigma}+5(n-1) \omega \\
& \leq-\frac{2 f\left(y_{1}\right)}{3 \sigma}+\frac{f\left(y_{1}\right)}{2 \sigma}+5(n-1) \omega \\
& <0 .
\end{aligned}
$$

Since $|\nabla \psi|,|\nabla \eta|$ are uniformly bounded the maximum principle [13, Theorem 10.1] implies that $\psi-\eta$ has no interior maximum. In particular,

$$
\begin{aligned}
\psi\left(y_{1}\right)-\varphi_{ \pm}\left(y_{1}\right)+2 \omega \varrho^{2} & =\psi\left(y_{1}\right)-\eta\left(y_{1}\right) \\
& \leq \sup _{\partial B_{\varrho}\left(y_{1}\right)}(\psi-\eta)=\omega \varrho^{2}
\end{aligned}
$$

and we deduce that

$$
\psi\left(y_{1}\right)-\varphi_{ \pm}\left(y_{1}\right)<0,
$$

which is a contradiction to (5.7). 
This shows by Assumption 4.2 and (4.10), (4.11) that

$$
f \leq 0 \text { if } N_{0} \geq 2 \text { and } u=1 \text { in }\left\{t>\varphi_{+}(y)\right\} .
$$

By a symmetry argument $u \mapsto-u$ it follows that

$$
f \geq 0 \text { if } N_{0} \geq 2 \text { and } u=-1 \text { in }\left\{t>\varphi_{+}(y)\right\} .
$$

Applying (5.11), (5.12) to the functions $(y, t) \mapsto u(y,-t),(y, t) \mapsto f(y,-t)$ we obtain that

$$
f \leq 0 \text { if } N_{0} \geq 3 \text { is odd and } u=-1 \text { in }\left\{t>\varphi_{+}(y)\right\}
$$

and that

$$
f \geq 0 \text { if } N_{0} \geq 3 \text { is odd and } u=1 \text { in }\left\{t>\varphi_{+}(y)\right\} .
$$

Putting together (5.11)-(5.14) and using (3.2) we deduce the conclusion (1), (2) of Theorem 3.2. The conclusion (3) follows from Proposition A.1, and the statement (4) is deduced from (1), (3) and (3.2).

\section{Proof of Proposition 5.2}

Assume that (5.7) does not hold, that is

$$
\sup _{B_{\varrho}\left(y_{1}\right)}\left(\varphi_{+}-\psi\right)>0
$$

By (5.4) and (5.6)

$$
\varphi_{+}<\psi \quad \text { on } \partial B_{\varrho}\left(y_{1}\right)
$$

Since $\psi$ is continuous and $\varphi_{+}$is upper-semicontinuous there exists for any $0<\varrho_{2}<\varrho$ a real number $0<\varrho_{3}<\varrho_{2}$ such that

$$
\begin{aligned}
& \sup _{B_{\varrho_{3}}\left(y_{1}\right)}\left(\varphi_{+}-\psi\right)>0, \\
& \varphi_{+}<\psi \quad \text { on } B_{\varrho}\left(y_{1}\right) \backslash B_{\varrho_{3}}\left(y_{1}\right) .
\end{aligned}
$$

As explained before we will use that $u_{\varepsilon}$ behaves as if the curvature of the sharp interface limit is given by $f / \sigma$, instead of $f /(N \sigma)$. In a first step we construct functions $v_{\varepsilon}$ such that

$$
\begin{aligned}
& -\varepsilon \Delta v_{\varepsilon}+\frac{1}{\varepsilon} W^{\prime}\left(v_{\varepsilon}\right) \leq \frac{7}{9} f\left(y_{1}\right), \\
& v_{\varepsilon} \rightarrow 2 \mathcal{X}_{\{(y, t): t>\psi(y)\}}-1 .
\end{aligned}
$$

In the second step we will apply a comparison principle to $u_{\varepsilon}, v_{\varepsilon}$ to obtain a contradiction in the limit $\varepsilon \rightarrow 0$.

\subsection{Construction of $v_{\varepsilon}$}

The two ingredients to construct $v_{\varepsilon}$ are a modified distance function from graph $(\psi)$ and the optimal profile and first order-correction of the one-dimensional minimization problem associated to the Cahn-Hilliard functional. 
Definition 6.1 We define

$$
M:=\operatorname{graph}\left(\psi\left\lfloor B_{\varrho}\left(y_{1}\right)\right)\right.
$$

and denote by $\mathrm{d}:=\operatorname{dist}(M, \cdot)$ the signed distance function from $M$, taken positive in the region 'above' $M$. Moreover we let $\Pi_{M}: \mathbb{R}^{n} \rightarrow M$ be the orthogonal projection onto $M$ and $\left(\kappa_{i}\right)_{i=1, \ldots, n-1}$ the principal curvatures of $M$. Finally we define for $x \in \mathbb{R}^{n},(y, t)=\Pi_{M}(x)$

$$
\tilde{\kappa}_{i}(x):=\kappa_{i}(y, t),
$$

which is well-defined in a neighborhood of $M$.

Remark 6.2 Since $\psi$ is smooth we deduce that $M$ is a smooth hypersurface and that there exists $\delta>0, \delta=\delta\left(\|\psi\|_{C^{2}\left(B_{\varrho}\left(y_{1}\right)\right)}, \varrho_{2}\right)$ such that the distance function $d$ is unique and smooth in a neighborhood

$$
G=\left\{x \in B_{\varrho_{2}}\left(y_{1}\right) \times\left(-5 \varrho_{0}, 5 \varrho_{0}\right):|\mathrm{d}(x)|<\delta\right\} .
$$

Moreover

$$
\Delta \mathrm{d}=-\sum_{i=1}^{n-1} \tilde{\kappa}_{i}+O(\mathrm{~d})=-\frac{2 f\left(y_{1}\right)}{3 \sigma}+O(\mathrm{~d}) .
$$

holds in $G$ [13, Lemma 14.17].

We turn to the optimal profile for the one-dimensional minimization in the Cahn-Hilliard energy.

Remark 6.3 Let $\phi_{0}: \mathbb{R} \rightarrow[-1,1]$ be the optimal profile, that is the solution of

$$
\begin{aligned}
& -\phi_{0}^{\prime \prime}+W^{\prime}\left(\phi_{0}\right)=0, \\
& \phi_{0}(-\infty)=-1, \quad \phi_{0}(+\infty)=1, \quad \phi_{0}(0)=0,
\end{aligned}
$$

and let $\phi_{1}: \mathbb{R} \rightarrow \mathbb{R}$ be the first order correction (see [26]),

$$
\begin{aligned}
& -\phi_{1}^{\prime \prime}+W^{\prime \prime}\left(\phi_{0}\right) \phi_{1}=-\phi_{0}^{\prime}+\sigma \\
& \phi_{1}( \pm \infty)=\frac{\sigma}{W^{\prime \prime}( \pm 1)}, \quad \phi_{1}(0)=0 .
\end{aligned}
$$

Since the distance function $d$ is smooth only in a neighborhood of $\operatorname{graph}(\psi)$ we have to modify the distance function.

Definition 6.4 For $\varepsilon>0$ we choose $\delta(\varepsilon)>0$ such that

$$
\delta(\varepsilon) \rightarrow 0, \frac{\delta(\varepsilon)}{\varepsilon} \rightarrow \infty \quad \text { as } \varepsilon \rightarrow 0
$$

and such that the conditions

$$
\frac{1}{\delta(\varepsilon)} \phi_{0}^{\prime}\left(\varepsilon^{-1} \delta(\varepsilon)\right), \frac{1}{\varepsilon} \phi_{0}^{\prime \prime}\left(\varepsilon^{-1} \delta(\varepsilon)\right) \rightarrow 0 \quad \text { as } \varepsilon \rightarrow 0
$$

are satisfied. Moreover we choose smooth functions $\beta_{\varepsilon}: \mathbb{R} \rightarrow \mathbb{R}, \varepsilon>0$, with

$$
\beta_{\varepsilon}(r)= \begin{cases}r & \text { for }|r| \leq \frac{\delta(\varepsilon)}{3} \\ -\delta(\varepsilon) & \text { for } r \leq-2 \delta(\varepsilon) \\ \delta(\varepsilon) & \text { for } r \geq 2 \delta(\varepsilon)\end{cases}
$$


such that

$$
\begin{aligned}
& 0 \leq \beta_{\varepsilon}^{\prime} \leq 1, \\
& 0 \geq \beta_{\varepsilon}^{\prime \prime} \geq-\frac{3}{\delta(\varepsilon)} .
\end{aligned}
$$

We then define the modified distance functions $d_{\varepsilon}$,

$$
d_{\varepsilon}(x):=\beta_{\varepsilon}(\mathrm{d}(x)) .
$$

Remark 6.5 We observe that by (6.13)

$$
\{|\mathrm{d}| \leq 2 \delta(\varepsilon)\} \subset G
$$

for all $\varepsilon \leq \varepsilon_{0}\left(\|\psi\|_{C^{2}\left(B_{\varrho}\left(y_{1}\right)\right)}\right)$ and deduce that $d_{\varepsilon}$ is smooth for sufficiently small $\varepsilon>0$. We compute that

$$
\begin{aligned}
\nabla d_{\varepsilon} & =\beta_{\varepsilon}^{\prime}(\mathrm{d}) \nabla \mathrm{d}, \\
\Delta d_{\varepsilon} & =\beta_{\varepsilon}^{\prime \prime}(\mathrm{d})+\beta_{\varepsilon}^{\prime}(\mathrm{d}) \Delta \mathrm{d} \\
& =\beta_{\varepsilon}^{\prime \prime}(\mathrm{d})-\beta_{\varepsilon}^{\prime}(\mathrm{d})\left(\frac{2 f\left(y_{1}\right)}{3 \sigma}+O(\mathrm{~d})\right),
\end{aligned}
$$

where we have used (6.8). For $\varepsilon<\varepsilon_{0}(\psi)$ we obtain that

$$
\left.\beta_{\varepsilon}^{\prime} \circ \mathrm{d}=0 \quad \text { in the set }\{|\mathrm{d}|>2 \delta(\varepsilon)\}\right\}
$$

and we deduce from (4.8), (6.16), (6.17) that

$$
\left|\Delta d_{\varepsilon}\right| \leq C\left(1+\frac{1}{\delta(\varepsilon)}\right) .
$$

We are now ready to define $v_{\varepsilon}$.

Definition 6.6 Let $\varepsilon_{0}=\varepsilon_{0}\left(\|\psi\|_{C^{2}\left(B_{\varrho}\left(y_{1}\right)\right)}\right)$ be chosen such that (6.19) holds. We then define $v_{\varepsilon}: B_{Q_{2}}\left(y_{1}\right) \times \mathbb{R} \rightarrow \mathbb{R}$,

$$
v_{\varepsilon}(x):=\phi_{0}\left(\varepsilon^{-1} d_{\varepsilon}(x)\right)+\varepsilon \phi_{1}\left(\varepsilon^{-1} d_{\varepsilon}(x)\right) \frac{2}{3 \sigma} f\left(y_{1}\right) .
$$

\subsection{Comparison of $u_{\varepsilon}, v_{\varepsilon}$}

\subsubsection{Subsolution property}

We are going to show that $v_{\varepsilon}$ is a suitable subsolution of a (diffuse) constant curvature equation.

We first compute that, using (6.11),

$$
\begin{aligned}
-\varepsilon \Delta v_{\varepsilon}=- & \frac{1}{\varepsilon} \phi_{0}^{\prime \prime}\left(\varepsilon^{-1} d_{\varepsilon}\right)\left|\nabla d_{\varepsilon}\right|^{2}-\phi_{0}^{\prime}\left(\varepsilon^{-1} d_{\varepsilon}\right) \Delta d_{\varepsilon} \\
& +\frac{2 f\left(y_{1}\right)}{3 \sigma}\left(-W^{\prime \prime}\left(\phi_{0}\left(\varepsilon^{-1} d_{\varepsilon}\right)\right) \phi_{1}\left(\varepsilon^{-1} d_{\varepsilon}\right)-\phi_{0}^{\prime}\left(\varepsilon^{-1} d_{\varepsilon}\right)+\sigma\right)\left|\nabla d_{\varepsilon}\right|^{2} \\
& -\varepsilon \phi_{1}^{\prime}\left(\varepsilon^{-1} d_{\varepsilon}\right) \frac{2 f\left(y_{1}\right)}{3 \sigma} \Delta d_{\varepsilon}
\end{aligned}
$$

and

$$
\frac{1}{\varepsilon} W^{\prime}\left(v_{\varepsilon}\right)=\frac{1}{\varepsilon} W^{\prime}\left(\phi_{0}\left(\varepsilon^{-1} d_{\varepsilon}\right)\right)+W^{\prime \prime}\left(\phi_{0}\left(\varepsilon^{-1} d_{\varepsilon}\right)\right) \frac{2 f\left(y_{1}\right)}{3 \sigma} \phi_{1}\left(\varepsilon^{-1} d_{\varepsilon}\right)+O(\varepsilon) .
$$


Using (6.22) we deduce from (6.24), (6.25) that

$$
\begin{aligned}
-\varepsilon \Delta v_{\varepsilon}+\frac{1}{\varepsilon} W^{\prime}\left(v_{\varepsilon}\right)= & -\frac{1}{\varepsilon} \phi_{0}^{\prime \prime}\left(\left|\nabla d_{\varepsilon}\right|^{2}-1\right)-\phi_{0}^{\prime}\left(\Delta d_{\varepsilon}+\frac{2 f\left(y_{1}\right)}{3 \sigma}\left|\nabla d_{\varepsilon}\right|^{2}\right) \\
& -\frac{2 f\left(y_{1}\right)}{3 \sigma} \phi_{1} W^{\prime \prime}\left(\phi_{0}\right)\left(\left|\nabla d_{\varepsilon}\right|^{2}-1\right) \\
& +\frac{2}{3} f\left(y_{1}\right)\left|\nabla d_{\varepsilon}\right|^{2}+o\left(\varepsilon \delta(\varepsilon)^{-1}\right) .
\end{aligned}
$$

Proposition 6.7 For all $0<\varepsilon<\varepsilon_{0}$, $\varepsilon_{0}=\varepsilon_{0}(\psi, W)$,

$$
-\varepsilon \Delta v_{\varepsilon}+\frac{1}{\varepsilon} W^{\prime}\left(v_{\varepsilon}\right) \leq \frac{7}{9} f\left(y_{1}\right) \text {. }
$$

Proof We check (6.27) in the different regions.

STEP1. In the region $\left\{|\mathrm{d}| \leq \frac{1}{3} \delta(\varepsilon)\right\}$ holds $\left|\nabla d_{\varepsilon}\right|=1, \beta_{\varepsilon}^{\prime} \circ \mathrm{d}=1, \beta_{\varepsilon}^{\prime \prime} \circ \mathrm{d}=0$, and we obtain from (6.21), (6.26) that

$$
\begin{aligned}
-\varepsilon \Delta v_{\varepsilon}+\frac{1}{\varepsilon} W^{\prime}\left(v_{\varepsilon}\right) & =\frac{2}{3} f\left(y_{1}\right)+O(\mathrm{~d})+o\left(\varepsilon \delta(\varepsilon)^{-1}\right) \\
& \leq \frac{2}{3} f\left(y_{1}\right)+O(\delta(\varepsilon))+o\left(\varepsilon \delta(\varepsilon)^{-1}\right) .
\end{aligned}
$$

Therefore (6.27) holds for $\varepsilon>0$ sufficiently small.

STEP2. In $\{\mathrm{d} \geq 2 \delta(\varepsilon)\}$ we obtain

$$
\begin{aligned}
-\varepsilon & \Delta v_{\varepsilon}+\frac{1}{\varepsilon} W^{\prime}\left(v_{\varepsilon}\right) \\
& =\frac{1}{\varepsilon} W^{\prime}\left(\phi_{0}\left(\varepsilon^{-1} \delta(\varepsilon)\right)+\varepsilon \phi_{1}\left(\varepsilon^{-1} \delta(\varepsilon)\right) \frac{2}{3 \sigma} f\left(y_{1}\right)\right) \\
& =\frac{1}{\varepsilon} W^{\prime}\left(\phi_{0}\left(\varepsilon^{-1} \delta\right)\right)+W^{\prime \prime}\left(\phi_{0}\left(\varepsilon^{-1} \delta\right)\right) \phi_{1}\left(\varepsilon^{-1} \delta\right) \frac{2}{3 \sigma} f\left(y_{1}\right)+O(\varepsilon) \phi_{1}\left(\varepsilon^{-1} \delta\right) .
\end{aligned}
$$

From (6.9), (6.14) and (6.12), (6.13) we deduce that (6.27) holds in $\{d \geq 2 \delta(\varepsilon)\}$ for sufficiently small $\varepsilon>0$. By similar calculations we obtain (6.27) also in the region $\{\mathrm{d} \leq-2 \delta(\varepsilon)\}$. STEP3. Let us now consider the set $\left\{\frac{1}{3} \delta(\varepsilon) \leq \mathrm{d} \leq 2 \delta(\varepsilon)\right\}$ and estimate the different terms in (6.26). We first obtain from (6.14) that in this region

$$
-\frac{1}{\varepsilon} \phi_{0}^{\prime \prime}\left(\varepsilon^{-1} d_{\varepsilon}\right)\left(\left|\nabla d_{\varepsilon}\right|^{2}-1\right) \rightarrow 0 \quad \text { as } \varepsilon \rightarrow 0 .
$$

Next we compute that, using (6.20), (6.21) and (6.17),

$$
\begin{aligned}
\mid- & \phi_{0}^{\prime}\left(\varepsilon^{-1} d_{\varepsilon}\right)\left(\Delta d_{\varepsilon}+\frac{2 f\left(y_{1}\right)}{3 \sigma}\left|\nabla d_{\varepsilon}\right|^{2}\right) \mid \\
& =\phi_{0}^{\prime}\left(\varepsilon^{-1} d_{\varepsilon}\right)\left|\beta_{\varepsilon}^{\prime \prime}(\mathrm{d})+\beta_{\varepsilon}^{\prime}(\mathrm{d})\left(\frac{-2 f\left(y_{1}\right)}{3 \sigma}+O(\mathrm{~d})\right)+\frac{2 f\left(y_{1}\right)}{3 \sigma} \beta_{\varepsilon}^{\prime}(\mathrm{d})^{2}\right| \\
& \leq \phi_{0}^{\prime}\left((3 \varepsilon)^{-1} \delta(\varepsilon)\right)\left(\frac{3}{\delta(\varepsilon)}+\frac{4 f\left(y_{1}\right)}{3 \sigma}+O(\delta(\varepsilon))\right) .
\end{aligned}
$$

Hence, by (6.14)

$$
-\phi_{0}^{\prime}\left(\varepsilon^{-1} d_{\varepsilon}\right)\left(\Delta d_{\varepsilon}+\frac{2 f\left(y_{1}\right)}{3 \sigma}\left|\nabla d_{\varepsilon}\right|^{2}\right) \rightarrow 0 \quad \text { as } \varepsilon \rightarrow 0 .
$$


Finally we observe that in $\left\{\frac{1}{3} \delta(\varepsilon) \leq \mathrm{d} \leq 2 \delta(\varepsilon)\right\}$

$$
a_{\varepsilon}:=\frac{\phi_{1}\left(\varepsilon^{-1} d_{\varepsilon}\right) W^{\prime \prime}\left(\phi_{0}\left(\varepsilon^{-1} d_{\varepsilon}\right)\right)}{\sigma}=1+o(1)
$$

by (6.12), (6.13) and we deduce for the last two lines in (6.26) that

$$
\frac{2}{3} f\left(y_{1}\right)\left(\left(-a_{\varepsilon}+1\right)\left|\nabla d_{\varepsilon}\right|^{2}+a_{\varepsilon}\right)=\frac{2}{3} f\left(y_{1}\right)+o(1) .
$$

We obtain from (6.26) and (6.29), (6.30), (6.32) that (6.27) holds in $\left\{\frac{1}{3} \delta(\varepsilon) \leq \mathrm{d} \leq 2 \delta(\varepsilon)\right\}$ for all $\varepsilon>0$ sufficiently small. By similar considerations we prove (6.27) also in $\{-\delta(\varepsilon) \leq$ $\left.\mathrm{d} \leq-\frac{1}{3} \delta(\varepsilon)\right\}$.

\subsubsection{Comparison in the bulk regions}

Lemma 6.8 As $\varepsilon \rightarrow 0$

$$
\begin{aligned}
& u_{\varepsilon} \rightarrow 1 \text { uniformly on each compact subset of }\left\{t>\varphi_{+}(y)\right\}, \\
& u_{\varepsilon} \rightarrow(-1)^{N_{0}} \quad \text { uniformly on each compact subset of }\left\{t<\varphi_{-}(y)\right\} .
\end{aligned}
$$

and

$$
\begin{aligned}
& v_{\varepsilon} \rightarrow 1 \quad \text { uniformly on each compact subset of }\{t>\psi(y)\}, \\
& v_{\varepsilon} \rightarrow-1 \quad \text { uniformly on each compact subset of }\{t<\psi(y)\} .
\end{aligned}
$$

Proof By [42, Proposition 4.2] either $u_{\varepsilon} \rightarrow 1$ or $u_{\varepsilon} \rightarrow-1$ uniformly on each compact subset of $\Omega \backslash \operatorname{supp} \mu$. By (4.11), (4.12) we conclude (6.33), (6.34). The construction of $v_{\varepsilon}$ yields (6.35), (6.36).

For $\varepsilon>0$ such that (4.8), (4.13) hold we deduce that

$$
f_{\varepsilon}>\frac{8}{9} f\left(y_{1}\right) \quad \text { in } B_{\varrho_{0}}(0) \times\left(-5 \varrho_{0}, 5 \varrho_{0}\right) .
$$

In fact, in this region we compute that

$$
\frac{f\left(y_{1}\right)}{f_{\varepsilon}} \leq \frac{\left|f\left(y_{1}\right)-f(0)\right|+f(0)}{f(0)-|f-f(0)|-\left|f_{\varepsilon}-f\right|} \leq \frac{28}{25}<\frac{9}{8} .
$$

Lemma 6.9 Choose $c_{0}>0$ such that

$$
W^{\prime \prime}(r) \geq \frac{W^{\prime \prime}(1)}{2}>0 \text { for all }|r| \geq 1-c_{0}
$$

and set $\Omega^{\prime}:=B_{\varrho_{0}}(0) \times\left(-5 \varrho_{0}, 5 \varrho_{0}\right)$.

Let $\lambda_{\varepsilon,+}, \lambda_{\varepsilon,-}$ be the solutions, close to +1 and -1 , respectively, of

$$
\frac{1}{\varepsilon} W^{\prime}\left(\lambda_{\varepsilon, \pm}\right)=\frac{8}{9} f\left(y_{1}\right)
$$

Then there exists for any bounded domain $U \subset \subset \Omega^{\prime}$ and any $k \in \mathbb{N}$ a constant $C_{k}=$ $C_{k}\left(n, U, \Omega^{\prime}\right)$ such that for all $\varepsilon<\varepsilon_{0}\left(k, c_{0}\right)$

$$
u_{\varepsilon} \geq \lambda_{\varepsilon,-}-C_{k} \varepsilon^{k} \quad \text { in } U
$$


Assume (6.38), (6.39), and in addition that there exists $\Omega^{\prime \prime} \subset \Omega^{\prime}$ such that

$$
u_{\varepsilon} \geq 1-c_{0} \quad \text { in } \Omega^{\prime \prime} \text {. }
$$

Then there exists for any bounded domain $U \subset \subset \Omega^{\prime \prime}$ and any $k \in \mathbb{N}$ a constant $C_{k}=$ $C_{k}\left(n, U, \Omega^{\prime \prime}\right)$ such that

$$
u_{\varepsilon} \geq \lambda_{\varepsilon,+}-C_{k} \varepsilon^{k} \quad \text { in } U
$$

Proof We first show the second conclusion. We deduce from (6.37), (6.39) that

$$
-\varepsilon \Delta\left(u_{\varepsilon}-\lambda_{\varepsilon,+}\right)+\frac{1}{\varepsilon}\left(W^{\prime}\left(u_{\varepsilon}\right)-W^{\prime}\left(\lambda_{\varepsilon,+}\right)\right) \geq 0 .
$$

Consider first bounded domains $\Omega_{1}, \Omega_{2}$ such that

$$
U \subset \Omega_{1} \subset \subset \Omega_{2} \subset \Omega^{\prime \prime} .
$$

Chose a cut-off function $\phi \in C_{c}^{\infty}\left(\Omega^{\prime \prime}\right)$ such that $0 \leq \phi \leq 1$ and

$$
\phi=1 \quad \text { in } \Omega_{1}, \quad \phi=0 \quad \text { in } \Omega^{\prime \prime} \backslash \Omega_{2} .
$$

Next we define

$$
\left(u_{\varepsilon}-\lambda_{\varepsilon,+}\right)_{-}:=\min \left(0, u_{\varepsilon}-\lambda_{\varepsilon,+}\right) \leq 0,
$$

we multiply (6.43) by $\left(u_{\varepsilon}-\lambda_{\varepsilon,+}\right)_{-} \phi^{2}$, and integrate over $\Omega_{2}$. We then deduce that

$$
\begin{aligned}
\int_{\Omega_{2}} \varepsilon\left|\nabla\left(u_{\varepsilon}-\lambda_{\varepsilon,+}\right)_{-}\right|^{2} \phi^{2} \leq & -\int_{\Omega_{2}} \varepsilon\left(u_{\varepsilon}-\lambda_{\varepsilon,+}\right)_{-} \nabla u_{\varepsilon} \cdot 2 \phi \nabla \phi \\
& -\int_{\Omega_{2}} \frac{1}{\varepsilon}\left(W^{\prime}\left(u_{\varepsilon}\right)-W^{\prime}\left(\lambda_{\varepsilon,+}\right)\right)\left(u_{\varepsilon}-\lambda_{\varepsilon,+}\right)_{-} \phi^{2} \\
\leq & \int_{\Omega_{2}} \varepsilon\left|\nabla\left(u_{\varepsilon}-\lambda_{\varepsilon,+}\right)_{-}\right|^{2} \phi^{2}+\int_{\Omega_{2}} \varepsilon|\nabla \phi|^{2}\left(u_{\varepsilon}-\lambda_{\varepsilon,+}\right)_{-}^{2} \\
& -\frac{1}{2 \varepsilon} W^{\prime \prime}(1) \int_{\Omega_{2}}\left(u_{\varepsilon}-\lambda_{\varepsilon,+}\right)_{-}^{2} \phi^{2},
\end{aligned}
$$

where in the last line we have used (6.38), (6.41). We therefore obtain that

$$
\frac{1}{2} W^{\prime \prime}(1) \int_{\Omega_{1}}\left(u_{\varepsilon}-\lambda_{\varepsilon,+}\right)_{-}^{2} \leq \varepsilon^{2}\|\nabla \phi\|_{L^{\infty}\left(\Omega_{2}\right)}^{2} \int_{\Omega_{2}}\left(u_{\varepsilon}-\lambda_{\varepsilon,+}\right)_{-}^{2} .
$$

Choosing now bounded domains $\Omega_{j}, j=1, \ldots, k+1$, such that

$$
U=\Omega_{1} \subset \subset \Omega_{2} \subset \subset \cdots \subset \subset \Omega_{k+1}=\Omega^{\prime \prime}
$$

and iterating the procedure above we deduce that

$$
\int_{U}\left(u_{\varepsilon}-\lambda_{\varepsilon,+}\right)_{-}^{2} \leq C\left(k, U, \Omega^{\prime \prime}\right) \varepsilon^{2 k} \int_{\Omega^{\prime \prime}}\left(u_{\varepsilon}-\lambda_{\varepsilon,+}\right)_{-}^{2} \leq C\left(k, U, \Omega^{\prime \prime}\right) \varepsilon^{2 k} .
$$

Assume now that for a $x_{1} \in U$

$$
u_{\varepsilon}\left(x_{1}\right)<\lambda_{\varepsilon,+}-c_{1} \varepsilon^{k},
$$


choose $r=r\left(U, \Omega^{\prime \prime}\right)$ such that $B_{r \varepsilon}\left(x_{1}\right) \subset \Omega^{\prime \prime}$, and consider the scaled functions $\tilde{u}, \tilde{f}$ : $B_{r}^{n}(0) \rightarrow \mathbb{R}$,

$$
\tilde{u}(x):=u_{\varepsilon}\left(x_{1}+\varepsilon x\right), \quad \tilde{f}(x):=f_{\varepsilon}\left(x_{1}+\varepsilon x\right) .
$$

Then $\tilde{u}, \tilde{f}$ satisfy the equation

$$
-\Delta \tilde{u}=-W^{\prime}(\tilde{u})+\varepsilon \tilde{f} .
$$

Since $u_{\varepsilon}$ is uniformly bounded by [42, Lemma 3.8] the right-hand side is uniformly bounded. We deduce by standard elliptic estimates that $\tilde{u} \in W^{2, q}\left(B_{r / 2}(0)\right)$ for all $1 \leq q<\infty$ and by the Sobolev inequality that

$$
\begin{aligned}
|\nabla \tilde{u}| \leq c_{2}(n, r) \quad \text { on } B_{r / 2}(0), \\
\left|\nabla u_{\varepsilon}\right| \leq \frac{c_{2}(n, r)}{\varepsilon} \quad \text { on } B_{\varepsilon r / 2}\left(x_{1}\right) .
\end{aligned}
$$

Thus (6.47) gives us for all $x \in B_{r \varepsilon^{k+1}}\left(x_{1}\right)$

$$
u_{\varepsilon}(x)=u_{\varepsilon}\left(x_{1}\right)+u_{\varepsilon}(x)-u_{\varepsilon}\left(x_{1}\right)<\lambda_{\varepsilon,+}-\left(c_{1}-c_{2}(n, r)\right) \varepsilon^{k}
$$

and we compute that for $c_{1}>c_{2}(n, r)$

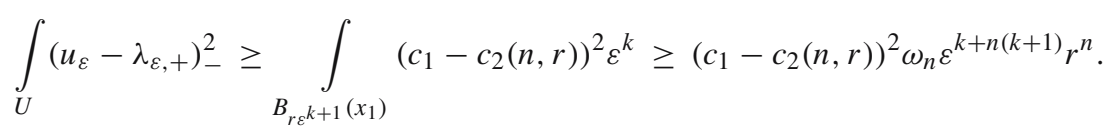

On the other hand, by (6.46) with $2 k$ replaced by $k+n(k+1)$ we obtain that

$$
\int_{U}\left(u_{\varepsilon}-\lambda_{\varepsilon,+}\right)_{-}^{2} \leq \tilde{C}\left(k, U, \Omega^{\prime \prime}\right) \varepsilon^{k+n(k+1)},
$$

which gives a contradiction for all $c_{1}=c_{1}\left(n, k, U, \Omega^{\prime \prime}\right)$ sufficiently large.

To prove (6.40) we first observe that

$$
-\varepsilon \Delta\left(-1+c_{0}\right)+\frac{1}{\varepsilon} W^{\prime}\left(-1+c_{0}\right) \geq \frac{8}{9} f\left(y_{1}\right)
$$

for all $\varepsilon>0$ sufficiently small. Since the minimum of two supersolutions is a supersolution we deduce that

$$
\tilde{u}_{\varepsilon}:=\min \left(u_{\varepsilon},-1+c_{0}\right)
$$

satisfies $\tilde{u}_{\varepsilon} \in W^{1,2}\left(\Omega^{\prime}\right)$ and

$$
\begin{aligned}
-\varepsilon \Delta \tilde{u}_{\varepsilon}+\frac{1}{\varepsilon} W^{\prime}\left(\tilde{u}_{\varepsilon}\right) & \geq \frac{8}{9} f\left(y_{1}\right), \\
\tilde{u}_{\varepsilon} & \leq-1+c_{0}
\end{aligned}
$$

Then we can prove by the same arguments as in the proof of (6.42) that

$$
\tilde{u}_{\varepsilon} \geq \lambda_{\varepsilon,-}-C_{k} \varepsilon^{k} \quad \text { in } U \text {. }
$$

Since $\lambda_{\varepsilon,-}-C_{k} \varepsilon^{k}<-1+c_{0}$ for $\varepsilon<\varepsilon_{0}(k)$ this proves (6.40).

We will employ a comparison principle on the cylinder $B_{\varrho_{2}}\left(y_{1}\right) \times\left(-4 \varrho_{0}, 4 \varrho_{0}\right)$. We first control the difference $u_{\varepsilon}-v_{\varepsilon}$ on the top and the bottom, starting with the following lemma. 
Lemma 6.10 Let $\lambda_{\varepsilon,+}, \lambda_{\varepsilon,-}$ be the solutions close to \pm 1 of (6.39) and let $\beta_{\varepsilon, \pm}$ denote the values of $v_{\varepsilon}$ 'away' from $M$,

$$
\begin{aligned}
& \beta_{\varepsilon,+}:=\phi_{0}\left(\varepsilon^{-1} \delta(\varepsilon)\right)+\varepsilon \phi_{1}\left(\varepsilon^{-1} \delta(\varepsilon)\right) \frac{2}{3 \sigma} f\left(y_{1}\right), \\
& \beta_{\varepsilon,-}:=\phi_{0}\left(-\varepsilon^{-1} \delta(\varepsilon)\right)+\varepsilon \phi_{1}\left(-\varepsilon^{-1} \delta(\varepsilon)\right) \frac{2}{3 \sigma} f\left(y_{1}\right) .
\end{aligned}
$$

Then there exists $\gamma>0, \varepsilon_{0}>0$ such that for all $0<\varepsilon<\varepsilon_{0}$

$$
\begin{aligned}
& \lambda_{\varepsilon,+}-\beta_{\varepsilon,+} \geq \gamma \varepsilon, \\
& \lambda_{\varepsilon,-}-\beta_{\varepsilon,-} \geq \gamma \varepsilon .
\end{aligned}
$$

Proof By a Taylor approximation

$$
\begin{aligned}
W^{\prime}\left(\beta_{\varepsilon, \pm}\right)= & W^{\prime}\left(\phi_{0}\left( \pm \varepsilon^{-1} \delta(\varepsilon)\right)\right)+W^{\prime \prime}\left(\phi_{0}\left( \pm \varepsilon^{-1} \delta(\varepsilon)\right)\right) \\
& \cdot \varepsilon \phi_{1}\left( \pm \varepsilon^{-1} \delta(\varepsilon)\right) \frac{2}{3 \sigma} f\left(y_{1}\right)+O\left(\varepsilon^{2}\right) \\
= & \frac{2}{3} f\left(y_{1}\right) \varepsilon+o(\varepsilon),
\end{aligned}
$$

where we have used that

$$
W^{\prime}\left(\phi_{0}\left( \pm \varepsilon^{-1} \delta(\varepsilon)\right)\right)=o(\varepsilon)
$$

by (6.9), (6.14) and that

$$
W^{\prime \prime}\left(\phi_{0}\left( \pm \varepsilon^{-1} \delta(\varepsilon)\right)\right) \phi_{1}\left(-\varepsilon^{-1} \delta(\varepsilon)\right) \frac{1}{\sigma} \rightarrow 1 \quad \text { as } \varepsilon \rightarrow 0
$$

by (6.12), (6.13).

We therefore deduce that

$$
\begin{aligned}
\left(\lambda_{\varepsilon, \pm}-\beta_{\varepsilon, \pm}\right) \int_{0}^{1} W^{\prime \prime}\left(s \lambda_{\varepsilon, \pm}+(1-s) \beta_{\varepsilon, \pm}\right) d s & =W^{\prime}\left(\lambda_{\varepsilon, \pm}\right)-W^{\prime}\left(\beta_{\varepsilon, \pm}\right) \\
& =\frac{2}{9} f\left(y_{1}\right) \varepsilon+o(\varepsilon) .
\end{aligned}
$$

Since $\lambda_{\varepsilon,-}, \beta_{\varepsilon,-}$ converge to -1 as $\varepsilon \rightarrow 0$ and since $W^{\prime \prime}(-1)>0$ we deduce from (6.52) that (6.51) holds for $\gamma>0$ and $\varepsilon>0$ sufficiently small. Analogously we obtain (6.50).

Proposition 6.11 For all $\varepsilon>0$ sufficiently small we obtain that

$$
u_{\varepsilon} \geq v_{\varepsilon} \text { in } \overline{B_{\varrho_{2}}\left(y_{1}\right)} \times\left[-4 \varrho_{0}, 4 \varrho_{0}\right]
$$

Proof Let us define the sets

$$
U:=B_{\varrho_{2}}\left(y_{1}\right) \times\left(-4 \varrho_{0}, 4 \varrho_{0}\right), \quad \Omega^{\prime}:=B_{\varrho}\left(y_{1}\right) \times\left(-5 \varrho_{0}, 5 \varrho_{0}\right) .
$$

Consider for $s>0$ the shifted functions $v_{\varepsilon}^{(s)}$,

$$
v_{\varepsilon}^{(s)}(y, t):=v_{\varepsilon}(y, t-s) \quad \text { for }(y, t) \in \Omega^{\prime},
$$

and the function

$$
\Phi(s):=\min _{\bar{U}}\left(u_{\varepsilon}-v_{\varepsilon}^{(s)}\right) .
$$


Assume now $\varepsilon<\varepsilon_{1}$, where we choose $\varepsilon_{1}>0$ below, and that (6.53) is not satisfied, hence

$$
\Phi(0)<0 .
$$

The definition of $v_{\varepsilon}$ in (6.23) implies that we can choose $s_{0}>0, s_{0}=s_{0}\left(\varrho_{0}\right)$ such that for all $\varepsilon>0$ sufficiently small

$$
v_{\varepsilon}^{\left(s_{0}\right)}=\beta_{\varepsilon,-} \text { in } \Omega^{\prime}
$$

Applying then Lemma 6.9 with $U, \Omega^{\prime}$ as above and $k=2$ we deduce that

$$
u_{\varepsilon} \geq \lambda_{\varepsilon,-}-O\left(\varepsilon^{2}\right) \text { in } U .
$$

Therefore (6.51) and (6.55), (6.56) imply that

$$
u_{\varepsilon}-v_{\varepsilon}^{\left(s_{0}\right)} \geq \lambda_{\varepsilon,-}-O\left(\varepsilon^{2}\right)-\beta_{\varepsilon,-} \geq \gamma \varepsilon-O\left(\varepsilon^{2}\right)
$$

and we deduce that

$$
\Phi\left(s_{0}\right)>\frac{\gamma}{2} \varepsilon
$$

for all $\varepsilon<\varepsilon_{1}$ and $\varepsilon_{1}>0$ chosen suitably small.

Since $\Phi$ is continuous (6.54), (6.57) imply the existence of $s_{*}>0$ and $x_{*} \in \bar{U}$ such that

$$
0=\left(u_{\varepsilon}-v_{\varepsilon}^{\left(s_{*}\right)}\right)\left(x_{*}\right)=\min _{\bar{U}}\left(u_{\varepsilon}-v_{\varepsilon}^{\left(s_{*}\right)}\right) .
$$

We first prove that $x_{*} \in U$. To this aim we consider the different parts of $\partial U$.

(1) For $\varepsilon_{1}>0$ chosen suitably small we have

$$
v_{\varepsilon}^{\left(s_{*}\right)}=\beta_{\varepsilon,-} \text { in } B_{\varrho_{2}}\left(y_{1}\right) \times\left(-9 \varrho_{0} / 2,-4 \varrho_{0}\right)
$$

and therefore, by Lemma 6.9 and Lemma 6.10,

$$
u_{\varepsilon} \geq v_{\varepsilon}^{\left(s_{*}\right)}+\frac{\gamma}{2} \varepsilon \quad \text { in } B_{\varrho_{2}}\left(y_{1}\right) \times\left(-9 \varrho_{0} / 2,-4 \varrho_{0}\right)
$$

for $\varepsilon<\varepsilon_{1}$ and $\varepsilon_{1}>0$ suitably small, see the argument above. This shows that

$$
x_{*} \notin \overline{B_{\varrho_{2}}\left(y_{1}\right)} \times\left\{-4 \varrho_{0}\right\} .
$$

(2) For $\varepsilon_{1}>0$ sufficiently small we obtain from Lemmas 6.8 and 6.9, applied to

$$
U_{+}=B_{\varrho_{2}}\left(y_{1}\right) \times\left(2 \varrho_{0}, 4 \varrho_{0}\right), \quad \Omega_{+}^{\prime \prime}=B_{\varrho}\left(y_{1}\right) \times\left(\varrho_{0}, 5 \varrho_{0}\right), k=2
$$

that

$$
u_{\varepsilon} \geq \lambda_{\varepsilon,+}-O\left(\varepsilon^{2}\right) \text { in } U_{+} .
$$

By (6.50) this implies that

$$
u_{\varepsilon} \geq \beta_{\varepsilon,+}+\gamma \varepsilon-O\left(\varepsilon^{2}\right) \geq \beta_{\varepsilon,+}+\frac{\gamma}{2} \varepsilon \geq v_{\varepsilon}+\frac{\gamma}{2}
$$

holds in $U_{+}$, hence

$$
x_{*} \notin \overline{B_{\varrho_{2}}\left(y_{1}\right)} \times\left\{4 \varrho_{0}\right\}
$$


(3) By (6.4) there exists bounded domains $U_{1}, \Omega_{1}, U_{2}$ such that

$$
\begin{aligned}
& U_{1} \subset \subset \Omega_{1} \subset \subset B_{\varrho}\left(y_{1}\right) \backslash \overline{B_{\varrho_{3}}\left(y_{1}\right)} \times\left(-5 \varrho_{0}, 5 \varrho_{0}\right) \\
& \Omega_{1} \subset \subset\left\{t>\varphi_{+}(y)\right\}, \\
& U_{2} \subset \subset\{t<\psi(y)\}, \\
& \partial B_{\varrho_{2}}\left(y_{1}\right) \times\left[-4 \varrho_{0}, 4 \varrho_{0}\right] \subset U_{1} \cup U_{2} .
\end{aligned}
$$

By similar arguments as above we first prove that $u_{\varepsilon}>v_{\varepsilon}^{\left(s_{*}\right)}$ in $U_{1}$. First we obtain from Lemma 6.8 that

$$
u_{\varepsilon} \geq 1-c_{0} \quad \text { in } \Omega_{1}
$$

and applying Lemmas 6.9 and 6.10 with $k=2$ we deduce that in $U_{1}$

$$
u_{\varepsilon} \geq \lambda_{\varepsilon,+}-O\left(\varepsilon^{2}\right)>v_{\varepsilon}^{\left(S_{*}\right)} .
$$

Since $s_{*}>0$ we obtain that for $\varepsilon<\varepsilon_{1}$, where $\varepsilon_{1}=\varepsilon_{1}\left(U_{2}, \varrho_{2}\right)$ is chosen sufficiently small,

$$
v_{\varepsilon}^{\left(s_{*}\right)} \leq \beta_{\varepsilon,-}+o(\varepsilon) \leq \lambda_{\varepsilon,-}-\gamma \varepsilon+o(\varepsilon)<u_{\varepsilon}
$$

in $U_{2}$. By (6.64), (6.65), (6.66) we deduce that

$$
u_{\varepsilon}>v_{\varepsilon}^{\left(s_{*}\right)} \quad \text { on } \partial B_{\varrho_{2}}\left(y_{1}\right) \times\left(-4 \varrho_{0}, 4 \varrho_{0}\right) .
$$

By (6.58)-(6.60), (6.67) we get that $u_{\varepsilon}-v_{\varepsilon}^{\left(s_{*}\right)}$ has an interior minimum with value zero at $x_{*}$. Using (2.3), (6.37), and (6.27) we therefore deduce that

$$
\begin{aligned}
0 & \geq-\varepsilon \Delta\left(u_{\varepsilon}-v_{\varepsilon}^{\left(s_{*}\right)}\right)\left(x_{*}\right) \\
& \geq f_{\varepsilon}\left(x_{*}\right)-\frac{7}{9} f\left(y_{1}\right)-\frac{1}{\varepsilon} W^{\prime}\left(u_{\varepsilon}\left(x_{*}\right)\right)+\frac{1}{\varepsilon} W^{\prime}\left(v_{\varepsilon}^{\left(s_{*}\right)}\left(x_{*}\right)\right) \\
& \geq \frac{1}{9} f\left(y_{1}\right) .
\end{aligned}
$$

This finally gives a contradiction and proves Proposition 6.11.

Proof of Proposition 5.2 By (6.23) we obtain that $v_{\varepsilon}(y, t) \rightarrow 1$ locally uniformly in $\{t>$ $\psi(y)$. Moreover, since $0<\varrho_{2}<\varrho$ was arbitrary (6.53) implies that $u_{\varepsilon} \geq v_{\varepsilon}$ in $B_{\varrho}\left(y_{1}\right)$. Therefore we deduce that

$$
\liminf _{\varepsilon \rightarrow 0} u_{\varepsilon} \geq 1 \quad \text { locally uniformly in }\left\{t>\psi(y), y \in B_{\varrho}\left(y_{1}\right)\right\} .
$$

Following the arguments in the proof of [42, Proposition 4.1] this implies that

$$
\operatorname{supp} \mu \cap\left\{t>\psi(y), y \in B_{\varrho}\left(y_{1}\right)\right\}=\emptyset .
$$

We therefore deduce from the definition of $\varphi_{+}$that (5.7) holds.

\section{Applications}

7.1 Stationary solutions for the Cahn-Hilliard equation

Consider the Cahn-Hilliard equation [7]

$$
\frac{\partial u_{\varepsilon}}{\partial t}=\Delta f_{\varepsilon}, \quad f_{\varepsilon}=-\varepsilon \Delta u_{\varepsilon}+\frac{1}{\varepsilon} W^{\prime}\left(u_{\varepsilon}\right)
$$


with Neumann boundary conditions for $u_{\varepsilon}$ and $f_{\varepsilon}$.

The stationary solutions of (7.1) are those with constant $f_{\varepsilon}$,

$$
-\varepsilon \Delta u_{\varepsilon}+\frac{1}{\varepsilon} W^{\prime}\left(u_{\varepsilon}\right)=\lambda_{\varepsilon}, \quad \lambda_{\varepsilon} \in \mathbb{R} .
$$

This is also the Euler-Lagrange equation of the volume constrained minimization problem for the Cahn-Hilliard energy (2.1),

$$
\inf \left\{E_{\varepsilon}(u): u \in H^{1,2}(\Omega), \int_{\Omega} u=m\right\} .
$$

To better understand stationary solutions of the Cahn-Hilliard equation the asymptotic of (7.2) is analyzed in a couple of papers. The behavior of (locally) energy minimizing solutions of $(7.2)$ is well understood $[16,19,21,40]$. In this case sequences $\left(u_{\varepsilon}\right)_{\varepsilon>0}$ with uniformly bounded energy converge to a constant-mean curvature hypersurface with single-multiplicity. This hypersurface is smooth except for a closed set of dimension at most $n-8$.

Solutions of the Cahn-Hilliard equation are observed to undergo pattern similar to unstable equilibria [14] and the behavior of general stationary points is another question of interest. However, this situation is more difficult due to the possibility of higher-multiplicity surfaces in the limit. Hutchinson-Tonegawa [16] showed that the limit is given by an integer-rectifiable varifold and that the weak mean curvature exists. However this mean curvature is only locally constant, the constant depending on the multiplicity. A higher multiplicity is also an obstacle to use Allard's regularity theory [3] and to obtain the smoothness of the limit.

As a corollary of Theorem 3.2 we can improve the previous results.

Theorem 7.1 Consider a sequence $\left(u_{\varepsilon}\right)_{\varepsilon>0} \subset H^{1,2}(\Omega)$ with a fixed volume constraint $\int_{\Omega} u_{\varepsilon}=m$, and a sequence $\left(\lambda_{\varepsilon}\right)_{\varepsilon>0} \subset \mathbb{R}$ such that (7.2) is satisfied. Suppose further that

$$
E_{\varepsilon}\left(u_{\varepsilon}\right) \leq \Lambda \text { for all } \varepsilon>0 \text {. }
$$

Then there exists a subsequence $\varepsilon \rightarrow 0$, a function $u \in B V(\Omega,\{-1,1\})$, and $\lambda:=$ $\lim _{\varepsilon \rightarrow 0} \lambda_{\varepsilon}$, such that $u_{\varepsilon} \rightarrow u$ in $L^{1}(\Omega)$. The phase boundary $\partial^{*}\{u=1\} \cap \Omega$ has constant mean curvature $\sigma H=\lambda$.

In the case that $\lambda \neq 0$ the phase boundary is up to a $\mathcal{H}^{n-1}$-nullset a smooth hypersurface. The energy measures $\mu_{\varepsilon}$ as defined in (2.6) converge to a measure $\mu$ that is up to the factor $2 \sigma$ integer-rectifiable, has constant mean curvature $\sigma H=\lambda$ and multiplicity one $\mathcal{H}^{n-1}$-almost everywhere on $\partial^{*}\{u=1\}$. Moreover 'hidden boundaries' can only occur in one phase and have zero mean curvature:

$$
\begin{aligned}
& \begin{cases}\operatorname{supp}(\mu) \cap\{u=1\}^{o}=\varnothing \quad \text { if } \lambda>0, \\
\operatorname{supp}(\mu) \cap\{u=-1\}^{o}=\varnothing \quad \text { if } \lambda<0,\end{cases} \\
& H=0 \quad \mathcal{H}^{n-1} \text {-almost everywhere on } \operatorname{supp}(\mu) \backslash \partial^{*}\{u=1\} .
\end{aligned}
$$

Proof It follows from [9, Lemma 3.4] that $\left|\lambda_{\varepsilon}\right| \leq c(m, \Lambda)$ and we may choose a subsequence such that $\lambda=\lim _{\varepsilon \rightarrow 0} \lambda_{\varepsilon}$ exists.

We therefore can apply Theorem 3.2 and obtain that there exists a subsequence $\varepsilon \rightarrow 0$ and limits $u, \mu$ of $u_{\varepsilon}, \mu_{\varepsilon}$. Moreover, $u \in B V(\Omega,\{-1,1\})$ and $(2 \sigma)^{-1} \mu$ is an integer-rectifiable varifold with weak mean curvature

$$
\sigma H=\lambda \mathcal{H}^{n-1}-\text { almost everywhere on } \partial^{*}\{u=1\} .
$$


Next it follows from Theorem 3.2 that $\lambda=0$ on the parts of $\partial^{*}\{u=1\} \cap \Omega$ with odd multiplicity larger than one, which shows that in the case $\lambda \neq 0$ the phase boundary is given as a constant curvature varifold with unit multiplicity. By Allard's regularity theory [3] we conclude the smoothness of the phase boundary. Finally (7.5), (7.6) follow from (3.4).

In general dimension we cannot insure good regularity of the hidden boundaries, due to the lack of regularity theory for general stationary integral varifold. Only for $n=2$ we can conclude that spt $\mu \cap K$ is given by straight line segments with possible junction points for all compact sets $K \subset \Omega \backslash \partial^{*}\{u=1\}$ [2].

\subsection{Critical points of the Ohta-Kawasaki functional}

The micro-phase separation of block copolymers exhibits the formation of complex patterns. Ohta-Kawasaki [25] and later Bahiana-Oono [4] used a phase-field like approach and proposed a free energy that is after a suitable rescaling given by

$$
F_{\varepsilon}(u)=\int_{\Omega}\left(\frac{\varepsilon}{2}|\nabla u|^{2}+\frac{1}{\varepsilon} W(u)+\frac{1}{2}|\nabla v|^{2}\right) d x,
$$

where $v(\cdot)=v[u](\cdot)$ is a solution of

$$
-\Delta v=u-\frac{1}{|\Omega|} \int_{\Omega} u \quad \text { in } \Omega, \quad \nabla v \cdot v_{\Omega}=0 \quad \text { on } \partial \Omega .
$$

The functional $F_{\varepsilon}$ extends the Cahn-Hilliard energy by a non-local term that describes longrange interactions between chains of macromolecules. For a derivation of this model by a density-functional approach see [11]. The set of (local) minimizers of $F_{\varepsilon}$ is extremely rich and (7.7) has drawn quite some attention [10,24,29,30]. The Gamma-limit of $F_{\varepsilon}$ as $\varepsilon \rightarrow 0$ and the convergence of the corresponding $H^{-1}$ gradient-flow that was proposed by Nishiura and Ohnishi [23] are also well-studied [12,15,28].

Critical points of $F_{\varepsilon}$ under a volume-constraint satisfy the Euler-Lagrange equation

$$
-\varepsilon \Delta u+\frac{1}{\varepsilon} W^{\prime}(u)+v[u]=\lambda,
$$

where $\lambda \in \mathbb{R}$ is a Lagrange-multiplier.

As a corollary of our results we obtain the convergence of stationary points of $F_{\varepsilon}$.

Theorem 7.2 Let $\Omega \subset \mathbb{R}^{n}$ be an bounded open set with Lipschitz boundary. Assume that we have a sequence $\left(u_{\varepsilon}\right)_{\varepsilon>0}$ such that

$$
F_{\varepsilon}\left(u_{\varepsilon}\right)+\left\|u_{\varepsilon}\right\|_{L^{\infty}(\Omega)} \leq \Lambda \text { for all } \varepsilon>0
$$

and such that

$$
-\varepsilon \Delta u_{\varepsilon}+\frac{1}{\varepsilon} W^{\prime}\left(u_{\varepsilon}\right)+v_{\varepsilon}=\lambda_{\varepsilon}
$$

holds for $\lambda_{\varepsilon} \in \mathbb{R}$ and the solutions $v_{\varepsilon}$ of

$$
-\Delta v_{\varepsilon}=u_{\varepsilon}-\frac{1}{|\Omega|} \int_{\Omega} u_{\varepsilon} \quad \text { in } \Omega, \quad \nabla v_{\varepsilon} \cdot v_{\Omega}=0 \quad \text { on } \partial \Omega, \quad \int_{\Omega} v_{\varepsilon}=0 .
$$

Then there exists a subsequence $\varepsilon \rightarrow 0$, a number $\lambda \in \mathbb{R}$, and a function $u \in$ $B V(\Omega,\{-1,1\})$ such that $\lambda=\lim _{\varepsilon \rightarrow 0} \lambda_{\varepsilon}$ and $u_{\varepsilon} \rightarrow u$ in $L^{1}(\Omega)$. Moreover $v_{\varepsilon} \rightarrow v$ in $C^{1, \alpha}(\bar{\Omega})$ for all $0<\alpha<1$ and $v$ solves (7.8). 
The energy measures $\mu_{\varepsilon}$ as defined in (2.6) converge to a measure $\mu$ that is up to the factor $2 \sigma$ integer-rectifiable and has a weak mean curvature that satisfies

$$
\sigma H= \begin{cases}-v+\lambda & \mathcal{H}^{n-1}-\text { almost everywhere on } \partial^{*}\{u=1\} \\ 0 & \mathcal{H}^{n-1}-\text { almost everywhere on } \operatorname{supp}(\mu) \backslash \partial^{*}\{u=1\} .\end{cases}
$$

Finally $\partial^{*}\{u=1\}$ has multiplicity one $\mathcal{H}^{n-1}$-almost everywhere in the set $\{v \neq \lambda\}$ and this part of the phase boundary is a $C^{3, \alpha}$-surface for all $\alpha<1$, except for a set of $\mathcal{H}^{n-1}$-measure zero.

Proof First we deduce from [9, Lemma 3.4] that the sequence $\left(\lambda_{\varepsilon}\right)_{\varepsilon>0}$ is uniformly bounded. By standard elliptic theory we obtain from (7.9), (7.11) that $v_{\varepsilon}$ is uniformly bounded in $W^{2, p}(\Omega)$ for all $1 \leq p<\infty$. Therefore Theorem 3.2 applies and we can repeat the arguments of the proof of Theorem 7.1. We omit the details here.

If $u_{\varepsilon}$ has in addition a local energy minimizing property for $F_{\varepsilon}$ we can draw stronger conclusions: Then $\mu$ has multiplicity one $\mu$-almost everywhere and is $C^{3, \alpha}$-smooth, see the arguments in [16].

Acknowledgments The research of Y. Tonegawa was partially funded by the Grant-in-aid for scientific research (B) No. 17340041. M. Röger thanks the Department of Mathematics, Hokkaido University Sapporo for their hospitality during his visit in August 2006. Y. Tonegawa thanks the Max Planck Institute for Mathematics in the Sciences, Leipzig for their hospitality during his visit in February 2007.

\section{Appendix A. A generalization of mean curvature to general phase boundaries}

Proposition A.1 (see [31, Proposition 3.1]) Let $\Omega \subset \mathbb{R}^{n}$ be open, $E \subset \Omega$, and $\mathcal{X}_{E} \in \mathrm{BV}(\Omega)$. Assume that there are two integral $(n-1)$-varifolds $\mu_{1}, \mu_{2}$ on $\Omega$ such that for $i=1,2$ the following hold:

$$
\begin{aligned}
& \partial^{*} E \subset \operatorname{supp}\left(\mu_{i}\right), \\
& \mu_{i} \text { has locally bounded first variation with mean curvature vector } \vec{H}_{\mu_{i}}, \\
& \vec{H}_{\mu_{i}} \in \mathrm{L}_{\mathrm{loc}}^{s}\left(\mu_{i}\right), s>n-1, s \geq 2 .
\end{aligned}
$$

Then

$$
\left.\vec{H}_{\mu_{1}}\right|_{\partial^{*} E}=\left.\vec{H}_{\mu_{2}}\right|_{\partial^{*} E}
$$

is satisfied $\mathcal{H}^{n-1}$-almost everywhere on $\partial^{*} E$.

This proposition justifies the following definition.

Definition A.2 Let $E \subset \Omega$ and $\mathcal{X}_{E} \in \mathrm{BV}(\Omega)$, and assume that there exists an integral $(n-1)$-varifold $\mu$ on $\Omega$ satisfying (A.1)-(A.3). Then we call

$$
\vec{H}:=\left.\vec{H}_{\mu}\right|_{\partial^{*} E}
$$

the generalized mean curvature vector of $\partial^{*} E$ and define a scalar mean curvature by

$$
H:=\vec{H} \cdot \frac{\nabla \mathcal{X}}{|\nabla \mathcal{X}|} \text { on } \partial^{*} E .
$$




\section{References}

1. Alikakos, N.D., Bates, P.W., Chen, X.: Convergence of the Cahn-Hilliard equation to the Hele-Shaw model. Arch. Rational Mech. Anal. 128(2), 165-205 (1994)

2. Allard, W.K., Almgren, F.J. Jr.: The structure of stationary one dimensional varifolds with positive density. Invent. Math. 34(2), 83-97 (1976)

3. Allard, W.K.: On the first variation of a varifold. Ann. Math. 95(2), 417-491 (1972)

4. Bahiana, M., Oono, Y.: Cell dynamical system approach to block copolymers. Phys. Rev. A 41(12), 6763-6771 (1990)

5. Brakke, K.A.: The motion of a surface by its mean curvature, volume 20 of Mathematical Notes. Princeton University Press, Princeton (1978)

6. Caginalp, G., Chen, X.: Convergence of the phase field model to its sharp interface limits. Eur. J. Appl. Math. 9(4), 417-445 (1998)

7. Cahn, J.W., Hilliard, J.E.: Free energy of a non-uniform system. I. interfacial energy. J. Chem. Phys. 28, 258-267 (1958)

8. Chen, X.: Generation and propagation of interfaces for reaction-diffusion equations. J. Differ. Equat. 96(1), 116-141 (1992)

9. Chen, X.: Global asymptotic limit of solutions of the Cahn-Hilliard equation. J. Differ. Geom. 44(2), 262-311 (1996)

10. Choksi, R.: Scaling laws in microphase separation of diblock copolymers. J. Nonlinear Sci. 11(3), 223-236 (2001)

11. Choksi, R., Ren, X.: On the derivation of a density functional theory for microphase separation of diblock copolymers. J. Stat. Phys. 113(1-2), 151-176 (2003)

12. Fife, P.C., Hilhorst, D.: The Nishiura-Ohnishi free boundary problem in the 1D case. SIAM J. Math. Anal. (Electronic) 33(3), 589-606 (2001)

13. Gilbarg, D., Trudinger, N.S.: Elliptic partial differential equations of second order. Classics in Mathematics. Springer, Berlin (2001)

14. Gurtin, M.E., Matano, H.: On the structure of equilibrium phase transitions within the gradient theory of fluids. Quart. Appl. Math. 46(2), 301-317 (1988)

15. Henry, M.: Singular limit of a fourth-order problem arising in the microphase separation of diblock copolymers. Adv. Differ. Equat. 6(9), 1049-1114 (2001)

16. Hutchinson, J.E., Tonegawa, Y.: Convergence of phase interfaces in the van der Waals-Cahn-Hilliard theory. Calc. Var. Partial Differ. Equat. 10(1), 49-84 (2000)

17. Ilmanen, T.: Convergence of the Allen-Cahn equation to Brakke's motion by mean curvature. J. Differ. Geom. 38(2), 417-461 (1993)

18. Luckhaus, S.: Solutions for the two-phase Stefan problem with the Gibbs-Thomson law for the melting temperature. Eur. J. Appl. Math. 1(2), 101-111 (1990)

19. Luckhaus, S., Modica, L.: The Gibbs-Thompson relation within the gradient theory of phase transitions. Arch. Rational Mech. Anal. 107(1), 71-83 (1989)

20. Luckhaus, S., Sturzenhecker, T.: Implicit time discretization for the mean curvature flow equation. Calc. Var. Partial Differ. Equat. 3(2), 253-271 (1995)

21. Modica, L.: The gradient theory of phase transitions and the minimal interface criterion. Arch. Rational Mech. Anal. 98(2), 123-142 (1987)

22. Modica, L., Mortola, S.: Un esempio di $\Gamma^{-}$-convergenza. Boll. Un. Mat. Ital. B (5) 14( 1), 285-299 (1977)

23. Nishiura, Y., Ohnishi, I.: Some mathematical aspects of the micro-phase separation in diblock copolymers. Phys. D 84(1-2), 31-39 (1995)

24. Ohnishi, I., Nishiura, Y., Imai, M., Matsushita, Y.: Analytical solutions describing the phase separation driven by a free energy functional containing a long-range interaction term. Chaos 9(2), 329-341 (1999)

25. Ohta, T., Kawasaki, K.: Equilibrium morphology of block copolymer melts. Macromolecules 19(10), 2621-2632 (1986)

26. Paolini, M.: A quasi-optimal error estimate for a discrete singularly perturbed approximation to the prescribed curvature problem. Math. Comp. 66(217), 45-67 (1997)

27. Pego, R.L.: Front migration in the nonlinear Cahn-Hilliard equation. Proc. Roy. Soc. Lond. Ser. A 422(1863), 261-278 (1989)

28. Ren, X., Wei, J.: On the multiplicity of solutions of two nonlocal variational problems. SIAM J. Math. Anal. (Electronic) 31(4), 909-924 (2000)

29. Ren, X., Wei, J.: On energy minimizers of the diblock copolymer problem. Interfaces Free Bound. 5(2), 193-238 (2003)

30. Ren, X., Wei, J.: Existence and stability of spherically layered solutions of the diblock copolymer equation. SIAM J. Appl. Math. (Electronic) 66(3), 1080-1099 (2006) 
31. Röger, M.: Solutions for the Stefan problem with Gibbs-Thomson law by a local minimisation. Interfaces Free Bound. 6(1), 105-133 (2004)

32. Röger, M.: Existence of weak solutions for the Mullins-Sekerka flow. SIAM J. Math. Anal. (Electronic) 37(1), 291-301 (2005)

33. Rowlinson, J.S.: Translation of J. D. van der Waals' The thermodynamic theory of capillarity under the hypothesis of a continuous variation of density. J. Stat. Phys. 20(2), 197-244 (1979)

34. Schätzle, R.: A counterexample for an approximation of the Gibbs-Thomson law. Adv. Math. Sci. Appl. 7(1), 25-36 (1997)

35. Schätzle, R.: Hypersurfaces with mean curvature given by an ambient Sobolev function. J. Differ. Geom. 58(3), 371-420 (2001)

36. Schätzle, R.: Quadratic tilt-excess decay and strong maximum principle for varifolds. Ann. Sc. Norm. Super. Pisa Cl. Sci. (5) 3(1), 171-231 (2004)

37. Simon, L.: Lectures on geometric measure theory, volume 3 of Proceedings of the Centre for Mathematical Analysis, Australian National University. Australian National University Centre for Mathematical Analysis, Canberra (1983)

38. Soner, H.M.: Convergence of the phase-field equations to the Mullins-Sekerka problem with kinetic undercooling. Arch. Rational Mech. Anal. 131(2), 139-197 (1995)

39. Soner, H.M.: Convergence of the phase-field equations to the Mullins-Sekerka problem with kinetic undercooling. Arch. Rational Mech. Anal. 131(2), 139-197 (1995)

40. Sternberg, P.: The effect of a singular perturbation on nonconvex variational problems. Arch. Rational Mech. Anal. 101(3), 209-260 (1988)

41. Tonegawa, Y.: Phase field model with a variable chemical potential. Proc. Roy. Soc. Edinburgh Sect. A 132(4), 993-1019 (2002)

42. Tonegawa, Y.: A diffused interface whose chemical potential lies in a Sobolev space. Ann. Sc. Norm. Super. Pisa Cl. Sci. (5) 4(3), 487-510 (2005) 